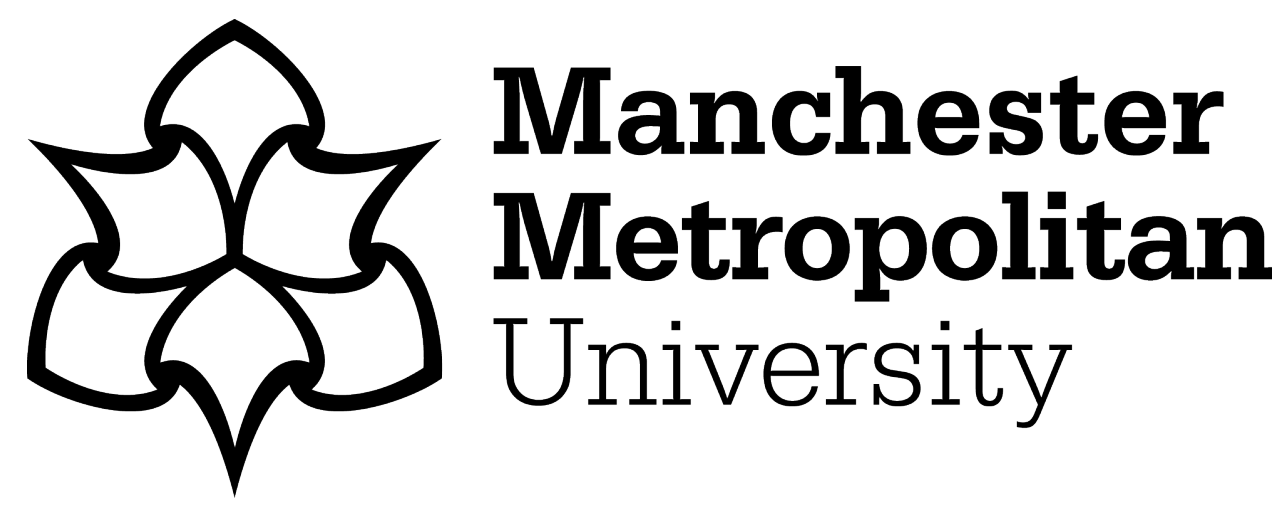

Venkatraman, Prabhuraj and Liauw, Chris (2018) Use of a carbon dioxide laser for environmentally beneficial generation of distressed/faded effects on indigo dyed denim fabric: Evaluation of colour change, fibre morphology, degradation and textile properties. Optics and Laser Technology, 111. pp. 701-713. ISSN 0030-3992

Downloaded from: https://e-space.mmu.ac.uk/621551/

Version: Accepted Version

Publisher: Elsevier

DOI: https://doi.org/10.1016/j.optlastec.2018.09.004

Usage rights: Creative Commons: Attribution-Noncommercial-No Derivative Works 4.0

Please cite the published version 


\title{
Use of a Carbon Dioxide Laser for Environmentally Beneficial Generation of Distressed/Faded Effects on Indigo Dyed Denim Fabric: Evaluation of Colour Change, Fibre Morphology, Degradation and Textile Properties
}

\author{
Praburaj D. Venkatraman ${ }^{1}$ and Christopher Mark Liauw ${ }^{2}$ \\ ${ }^{1}$ Manchester Fashion Institute, Faculty of Arts and Humanities and \\ ${ }^{2}$ School of Healthcare Science, Faculty of Science and Engineering, \\ Manchester Metropolitan University, Cavendish Street, Manchester M15 6BG
}

\begin{abstract}
Denim garments are particularly popular with the younger population of adults. Distressed or worn out effects have been and will continue to be popular with this market sector. These faded or worn effects have been achieved using a range of physical, chemical and mechanical finishes. Both wet and dry finishing of denim fabrics and garments pose severe environmental and health risks. Recently, environmentally beneficial decolourisation/ablation methods for denim fabrics have been investigated. Such methods have included plasma, laser, and ozone treatments. Researchers in this field have highlighted the potential of $\mathrm{CO}_{2}$ laser treatment of $100 \%$ cotton denim, however the textile performance post-treatment has not been properly investigated. In this study, light, medium and heavy weight indigo dyed $100 \%$ cotton denim fabrics were exposed to a $\mathrm{CO}_{2}$ laser at a range of power and intensity levels. Colour change was investigated using a Spectrophotometer, morphological structural analysis was carried using Scanning Electron Microscopy, and attenuated total reflectance Fourier transform Infrared spectroscopy (ATR-FTIR) was used to monitor the loss of indigo dye and degradation of the cellulose fibres. The thermal-oxidative degradation behaviour of fabrics was also studied using differential scanning calorimetry to obtain oxidation onset temperature. In addition, several fabric performance assessments were carried to evaluate tensile strength, colour fastness to washing, air permeability and thickness. Findings reveal that the grayscale rating, which is the tone density and hence laser power affected the colour change and as the grayscale increased, the colour fading was higher and affected the fabric performance across all fabric weights. Based on this, the research recommends an optimum set of laser processing parameters to produce stressed or faded denim effects without compromising the fabric performance. This research demonstrates that faded effects on denim can be produced with low environmental and health risks.
\end{abstract}

Key words: Laser patterning, Denim, Indigo, Colour measurement, FTIR, Mechanical properties.

Corresponding author: p.venkatraman@mmu.ac.uk Manchester Fashion Institute, Manchester Metropolitan University, Manchester M15 6BG, UK 


\section{Highlights}

1. Faded effects on denim produced using a commercial laser cutting/engraving system

2. The process is more precise than established fading methods and is chemical free

3. Correct implementation of laser treatment ensures retention of fabric properties

4. Significant loss of blue colour is attainable with minimal fibre degradation

5. The laser process is reproducible and has the potential to produce complex designs

\section{Introduction}

Denim is a twill woven fabric and is popular among people of all ages. It is durable, strong and resists wear and tear due to its fabric structure. It is a woven fabric with dyed warp yarns and white coloured weft yarns. It is a widely manufactured fabric involving complex processes and machinery. In order to accrue green credentials, industry has adopted a plethora of environmentally sustainable methods, most particularly at the finishing stages (Paul, 2015). Dry finishing techniques are traditionally used to produce a range of effects including distressed / worn finishes in which the indigo (or other) dye is removed from the fibres. Many of these processes require a number of steps and are thus time consuming and arduous. A typical dry finishing process involves abrading the surface of the garment and the dust produced can potentially affect the health of the operator. The current demand for finished denim goods continues to increase over the years; the market value of the denim industry is expected to grow from $\$ 113$ to $\$ 153$ billion (Textile Magazine, 2016). Faded, distressed, or torn denim jeans are particularly popular with the younger population; skinny jeans are a typical example of a product requiring such finishes. In the recent years, many researchers (Jucienè et al. 2014 and Kan, 2014) have explored the use of lasers to partially remove surface colour and determine important parameters that affect colour change. Other forms of surface treatment such as corona discharge (Nourbakhsh and Yazdanshenas, 2008) and plasma (Ghoranneviss et al., 2007) have also been explored.

A laser is an energy source whose intensity and power can be precisely controlled (Dowden, 2009). Laser beams are widely used to cut various materials from metal to fabric; garment decoration using laser etching and engraving have grown in popularity to produce faded effects including surface textures and patterns. The applications of lasers in apparel manufacturing are diverse and include: fabric fault detection, fabric cutting, objective evaluation of seam pucker, accurate body measurements, laser fabric fading, engraving, laser welding of fabrics, bar code scanning and laser marking (Nayak and Padhye, 2016). Jeanologia launched a laser fading 
technique for denim garments that has replaced conventional methods of whiskering, sand blasting and other artisan techniques for creation of denim wear effects (Garcia, 2015).

The use of lasers to partially remove indigo dye from denim fabric surfaces (and thus produce faded effects) had been widely reported (Juciene et al. 2014, Kan et al. 2010, 2014). Three different types of pulsed lasers were investigated by Ortiz-Morales et al. (2003), including a CW CO2 laser ( $\square=10.62 \mu \mathrm{m})$, electro-optical pulsed laser Nd:YAG laser $(\square=1.06 \mu \mathrm{m})$ and CTH:YAG pulsed laser $(\square=2.09 \mu \mathrm{m})$. The researchers also evaluated colourfastness, tear strength, strength resistance and dimensional changes. Among the three types of laser, the CO2 laser fading method was identified as being highly efficient in terms of low heat generation and inherently lower cost due to simpler cooling systems. It was also suggested that low energy treatments resulted in marginal improvements in tear strength and shrinkage, relative to the pristine material. Laser power density and laser energy per unit area were found to be the key parameters affecting the level of fading. The levels of fading obtainable matched those produced by conventional methods. Ozguney (2009) reported the use of a CO2 laser beam $(10.6 \mu \mathrm{m})$ for fading denim fabrics and evaluated colour and mechanical properties. It further added for colour fading and mechanical properties $100-150 \mu$ s pulse times were suitable.

Juciene et al. (2014) reported that laser power and laser energy density were important parameters that influence $\Delta \mathrm{E}$ (change in fabric colour determined spectroscopically using CIE LAB / HSB colour models). They also noted colour change effects were different in warp and weft direction of denim fabric and inferred this observation was due to the laser beam falling in lengthwise and crosswise directions. In addition, SEM images indicated degradation of the textile fibres. Ozguney (2007) compared laser treated denim fabrics with those that had been pigment printed; the laser technique was more reproducible, and the desired effects are produced without additional processing such as drying and fixation. In addition, laser patterned samples had good dry and wet rubbing colourfastness compared to pigment printed samples. Tarhan and Sariisik (2009) concluded that $\mathrm{CO}_{2}$ laser treatment of textile materials is a viable alternative to conventional wet treatments such as stone washing, sand washing and bleaching for obtaining worn / distressed effects.

Ondogan et al. (2005) also investigated various laser design settings, evaluated fabric strength on 20 manually treated (sand blasting and abrasion by emery stone) and 20 laser treated denim trousers. They reported that the tensile strength of laser treated trousers was 10-30\% greater than manually patterned / finished trousers. They also found that computer control of the laser patterning process was very precise and consistent. Additionally, none of the wear or deformation associated with conventional fading processes (sanding and stoning) was observed using laser patterning. 
Kan et al. (2010 and 2014) demonstrated that, under the correct conditions, $\mathrm{CO}_{2}$ laser treatment is able to produce faded effects on denim fabric within short time periods. Dascalu et al. (2000) reported that removal of indigo dye from denim fabric after conducting experiments with three different laser wavelengths, power density and laser fluency. They added that $\mathrm{CO}_{2}$ laser affected fibres due to vaporisation. Exposed material reached its vaporisation stage rapidly and diffuses into atmosphere without interaction with laser and thermal degradation occurred on fabric surface. Ferrero et al. (2002) investigated the surface degradation of linen fabrics using laser exposure. The degradation, observed using Fourier transform infrared spectroscopy (FTIR) and differential scanning calorimetry (DSC), was compared with that obtained using an electron beam and a heat source. SEM imaging revealed localised surface degradation in the form of sponge like structures on the fibre surface; these were formed due to evolution of gaseous degradation products. FTIR revealed significant changes to the structure of the fibres including: broadening of various $\mathrm{C}-\mathrm{O}$ absorptions (indicating a loss of structural order), a reduction in the amount of adsorbed water and possible generation of carbonyl species as a result of oxidation (Ferrero et al. (2002), Chung et al. (2004)).

Štěpánková et al. (2011) reported the colour change effects on cotton twill fabric with vat dyes and exposed the fabric to infrared laser light. Colour measurements, surface degradation, SEM microscopic analysis and fabric tensile strength data were reported. These researchers claim that laser fluency was one of the parameters that influenced colour change and fabric strength. High and medium laser fluency affected fabric strength in weft direction. Medium laser fluency $7.8 \mathrm{~mJ} \mathrm{~cm}^{-2}$ affected colour change with less surface damage to fibres and added that vat dyes protected fabrics from laser light. Kan (2014a) confirmed that $\mathrm{CO}_{2}$ laser treatment is a clean process for denim fading applications. Various laser processing parameters including, laser power, laser resolution and pixel time were investigated and colour assessment of fabrics (torque free low twist and ring spun yarn) were reported. Colour fading was carried out using a $\mathrm{CO}_{2}$ laser and comparisons were made with cellulase treatment. Measured parameters included reflectance, colour yield (K/S values) and CIE L* $\mathrm{a}^{*} \mathrm{~b}^{*}$ values. Comparison of the colour yield data revealed that the laser method could offer a greater colour fading effect and was more controllable than the cellulase method. The conventional wet treatment method requires extensive rinsing with water and is therefore much more time consuming than the laser treatment method. Hence, the researchers concluded that laser method was cleaner as it saved energy, time and chemicals. Yuan et al., (2012), evaluated decorative laser patterning of 100\% polyester fabrics. They reported that laser resolution (dpi) and residence time $(\mu \mathrm{s})$ were important parameters to optimise and control; higher laser energies would lead to excessive melting and degradation. Ideal parameters were in between $30 \mathrm{dpi} / 120 \mu \mathrm{s}$ and $50 \mathrm{dpi} / 270 \mu \mathrm{s}$. The input voltage was reported to be $100 \mathrm{~V}$ ( $280 \mathrm{~W}$ in power). 
It should be noted that previous studies were deficient in that the effect of laser light on colour change over a range of different fabric parameters including, weight, fabric density and thickness had not been considered. In addition, previous studies have not considered the effect of laser power density on fabric performance, particularly surface morphology, fabric strength and durability, thermal degradation and analytical assessment using infrared spectroscopy. In addition, the conclusions/findings of previous studies were limited to one type of denim fabric and physical parameters of fabrics were not related to colour assessments.

\section{Aims and Objectives}

The primary purpose of this paper is to determine the optimum set of laser parameters for controlled decolourisation of indigo dye denim fabrics of varying density. A commercial $\mathrm{CO}_{2}$ laser-based cutting / patterning / etching system was used in this study. As well as monitoring of colour change, structure variations, thermal degradation, performance properties including fabric strength, colourfastness, and air permeability were examined via a range of methods including colourimetry, standard mechanical testing, FTIR, DSC and SEM imaging. This paper will provide a uniquely comprehensive understanding of how laser treatment parameters affect denim fabrics of varying density and thickness. Optimal treatment parameters will be provided along with monitoring of loss of colour and degradation using FTIR and DSC.

\section{Methods and Materials}

Fabric samples. Indigo dyed, 100\% cotton denim fabric samples of three different weights (Table 1) were chosen, supplied by Rofinor Texteis, Portugal. The twill fabrics had $2 / 1$ fabric structure (Figure 1).

Colour measurement. All fabrics were conditioned in standard laboratory conditions $20 \pm 2^{\circ} \mathrm{C}$ and relative humidity $65 \%$ for 24 hrs prior to the test. Fabric surface colour change, $(\Delta \mathrm{E}), \mathrm{CIE}$ $L^{*} a^{*} b^{*}$, HSB (Colour Hue, (H) Saturation, (S) and Brightness, (B)) and K/S values have been evaluated using spectrophotometer (SF600 DCI Spectraflash from Datacolour). Colour change was evaluated using the D65 illuminant and $10^{\circ}$ standard observer within the visible spectrum 400 to $700 \mathrm{~nm}$. Colour spaces are colour models that provide numeric formulations for defining, naming, and reproducing exact colour matches for electronic input and output devices such as a computer or printer (Bubonia, 2017). The K/S values

$$
\frac{K}{S}=\frac{(1-R)^{2}}{2 * R}
$$

Equation 1 represents Kubleka-Munk formula - where: $\mathrm{R}$ is the reflection value in maximum absorption wavelength; K - Absorption coefficient; S- scattering coefficient (Nobbs, 1985). 
The colour space $\mathrm{L}^{*} \mathrm{a}^{*} \mathrm{~b}^{*}$ was developed by CIE, Commission Internationale d'Eclairage (www.cie.co.at) to have numerical values and define exact colour when viewing in an output device. $\Delta \mathrm{E}$ changes to fabric colour that depends on parameters ( $\mathrm{L}, \mathrm{a}$, and $\mathrm{b}$ )

Where: $\Delta \mathrm{L}$ changes in lightness of the colour

$a^{*}$ - which indicates position between red and green (positive values designate red, negative values designate green); $a^{*}$ indicates redness or greenness of a sample; a negative $a^{*}$ value indicates the greener shade; whilst a positive a* indicates dark redness in the sample.

$\mathrm{b}^{*}$ - which indicates position between yellow and blue (positive values designate yellow and negative value blue)

$\mathrm{L}^{*}$ indicates lightness of the sample; higher the value the lighter the shade

B - colour brightness - measured from $0-100 \%$ (the most bright colour) (www.cie.co.at)

Colour Hue $\left(\mathrm{H}^{\circ}\right)$ defines colour itself whose value runs from $0-360^{\circ}$ from red- yellow- greenblue-purple-red and including all intermediary colours (Adobe Technical Guide). The HSB colour model is similar to Munsell system with three defining aspects, Hue, value and chroma.

Colour saturation $(\mathrm{S})$ indicates the degree to which the hue differs from natural gray, the value runs from $0 \%$ - no saturation to $100 \%$ full saturation (Adobe Technical Guide).

Standard performance tests. Various physical tests were carried out to evaluate physical and performance parameters in accordance with the British Standards. These include, fabric thickness (BS EN ISO 5084:1997), tensile strength (BS EN ISO 13934-1: 1999), air permeability (BS EN ISO 9237:1995) and colourfastness to washing (BS EN ISO 105 C10 2007). Air permeability was measured using Shirley Air Permeability tester. The pressure drop was maintained at $100 \mathrm{~Pa}\left(10 \mathrm{~mm}\right.$ head of water column) and test surface was $5.0 \mathrm{~cm}^{2}(25.4$ $\mathrm{mm}$ diameter). Note that the percentage relative tensile strength was used to decouple the effect of fabric thickness from laser induced fibre damage. Percentage relative tensile strength was determined using:

$$
\% \text { Relative tensile strength }\left(\% \overline{T S}_{\text {Rel }}\right)=\left[\frac{\overline{T S}_{\text {Sample }}}{\overline{T S}_{\text {Pristine }}}\right] \times 100
$$

Where: $\overline{T S}_{\text {Sample }}$ is the average tensile strength of the given laser etched sample and $\overline{T S}_{\text {Pristine }}$ is the average tensile strength of the respective pristine denim. Errors in $\% \overline{T S}_{R e l}$ were determined via quadrature addition:

$$
\Delta \% \overline{T S}_{\text {Rel }}=\left[\sqrt{\left(\frac{\Delta \overline{T S}_{\text {Sample }}}{\overline{T S}_{\text {Sample }}}\right)^{2}+\left(\frac{\Delta \overline{T S}_{\text {Pristine }}}{\overline{T S}_{\text {Pristine }}}\right)^{2}}\right] x \% \overline{T S_{\text {Rel }}}
$$


(Instructional Physics laboratory - Harvard, 2007).

Microscopy. A Carl Zeiss: Supra 40 VP scanning electron microscope (SEM) operated under variable pressure, was used to investigate the fabric morphology and observe degradation. Accelerating voltage was typically $20 \mathrm{kV}$ and working distance typically $6 \mathrm{~mm}$. A handheld 'DinoLite' light microscope was also used to obtain low magnification (ca. 50.5x) images of the samples for observation of the fabric design pattern.

Oxidation onset temperature. A Perkin Elmer DSC-7 was used for this analysis. A small fabric sample of $8.5 \mathrm{mg}$ was sealed in aluminium pan. Each sample was heated from $25^{\circ} \mathrm{C}$ to $450^{\circ} \mathrm{C}$ at $20^{\circ} \mathrm{C} / \mathrm{min}$ in oxygen $(30 \mathrm{~mL} / \mathrm{min})$. For this study, the oxidation onset temperature was defined as the temperature at the intersection of the baseline tangent and the tangent to the heat flow data where the rate of degradation was maximum.

Fourier transform Infrared (FTIR) spectroscopy. FTIR is a technique based on the unique vibration frequencies and vibration modes of covalent bonds in a molecule. It is obtained by passing infrared radiation through a sample of fabric and determining what fraction of incident radiation is absorbed at a particular energy (Stuart 2009). The FT-IR spectra were recorded from $4000 \mathrm{~cm}^{-1}$ to $450 \mathrm{~cm}^{-1}$ using Perkin Elmer Spectrum 2 fitted with a single bounce attenuated total reflectance (ATR) accessory ( 4 scans and resolution of $4 \mathrm{~cm}^{-1}$ ). The internal reflection element in the latter is diamond.

Laser patterning of denim samples. A Universal Laser ILS $12.57 \mathrm{CNC} \mathrm{CO}_{2}$ laser cutting / patterning / engraving system was used to pattern the denim samples. The wavelength of the laser used was $10.62 \mu \mathrm{m}$ and the power range available was $10 \mathrm{~W}$ to $75 \mathrm{~W}$, the pulsed wave mode was used throughout the study. A vector design was produced using Corel Draw and was transferred to the Universal Laser control software. A grayscale rating from $0 \%$ to $100 \%$ was used to depict the shade variations that is expected in a denim garment. The vector design $100 \%$ grayscale indicates higher tone density compared to $10 \%$ where there is less tone density. Various laser design parameters are selected before engraving the fabric with laser. In this study, two different laser power variations were chosen, $46 \mathrm{~W}(100 \%)$ and $23 \mathrm{~W}(50 \%)$ and was run at $100 \%$ speed. The laser engraving was carried out in the weft direction such that denim fading occurs more on the warp yarns and fabric thickness was also taken into consideration. The pulses per inch, which indicates the closeness of the laser pulse irradiation was maintained at 500 ppi (pulses per inch) for all the test samples. 
(a)
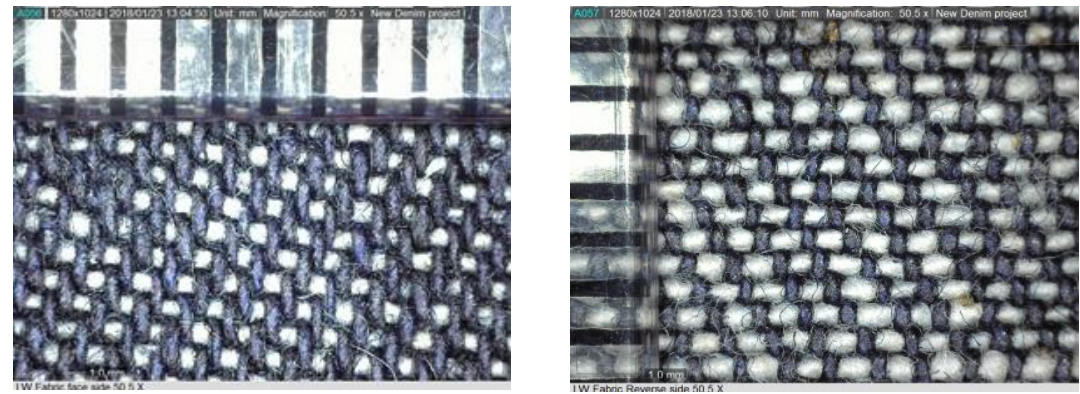

(b)
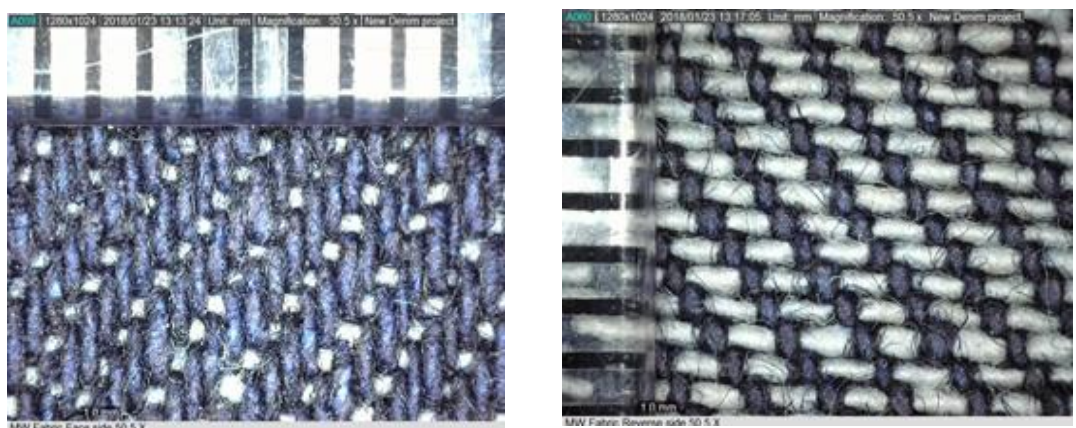

(c)

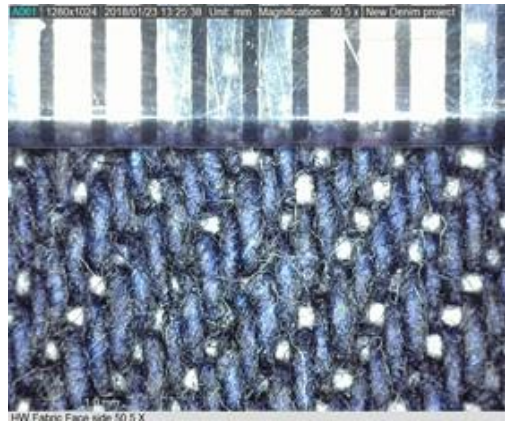

Face side

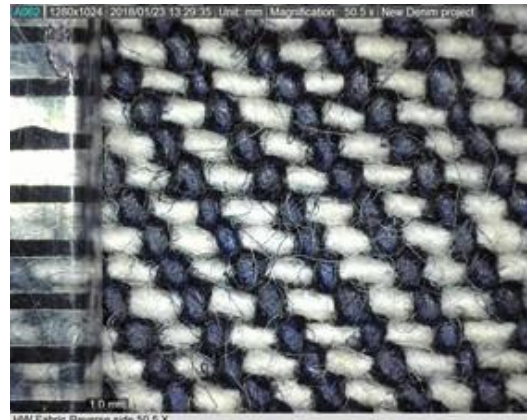

Reverse side

Figure 1. Micrographs of denim fabric samples (a) LW; (b) MW and (c) HW fabric. Ruler markings are $1 \mathrm{~mm}$

\section{Results and discussions}

The basic physical characteristics, including cover factor and density, of the light weight (LW), medium weight (MW) and heavy weight (HW) denim fabrics are provided in Table 1. Light micrographs showing the technical face and reverse side for each fabric type are given in Figure 2. Table 2 provides tensile strength of fabrics in warp and weft direction, it was evident that the HW fabric has higher strength compared LW and MW fabrics. Percentage elongation in warp direction was higher for HW fabric compared to LW and MW fabrics due to its higher density, and lower warp yarn count. Air permeability was higher for LW fabric compared to MW and HW fabrics. The colourfastness to rubbing of all the fabrics revealed that warp wet rubbing losses colour more than dry rubbing. Colourfastness to rubbing was tested to find out if the denim fabric could resist colour migration due to rubbing action and whether dyes are 
fixed adequately, as most denim fabrics are manufactured to lose its colour due to poor dyeing technique. It is normally assessed using grayscale rating, where 5 is excellent colourfastness and 1 being poor fastness. The fabrics demonstrated that under dry condition, the colourfastness was good, this was important when using laser engraving. Bulk density is the weight per volume of the fabric and is used to express the amount of thickness in a fabric due to air and the amount due to fibre, generally expressed in grams $/ \mathrm{cm}^{3}$ by measuring the weight of known fabric dimension and thickness (Watkins, 1995). MW and HW fabric has marginally higher bulk density compared to LW fabric. Fabrics with low density values will have good insulation. Cover factor indicates the extent to which area of a fabric is covered by a set of yarns, in other words the relative closeness of yarns within a fabric structure and it could be noted LW fabric had higher cover factor in warp direction compared to MW and HW samples, whilst lower fabric coverage in weft direction.

Table 1. Fabric properties

\begin{tabular}{|c|l|c|c|c|}
\hline & \multicolumn{1}{|c|}{ Fabric parameter/fabric type } & LW & MW & HW \\
\hline 1 & Fabric weight $\left(\mathrm{g} / \mathrm{m}^{2}\right)$ & $285( \pm 5.0)$ & $383( \pm 3.05)$ & $465( \pm 1.52)$ \\
\hline 2 & Thickness $(\mathrm{mm})$ & $0.66( \pm 0)$ & $0.84( \pm 0)$ & $1.10( \pm 0)$ \\
\hline 3 & Bulk density $\left(\mathrm{g} / \mathrm{cm}^{3}\right)$ & $0.43( \pm 0.01)$ & $0.45( \pm 0.0)$ & $0.45( \pm 0.0)$ \\
\hline 4 & Warp yarn count (tex) & 35 & 56 & 82 \\
\hline 5 & Weft yarn count (tex) & 46 & 87 & 108 \\
\hline 6 & Fabric structure & $86 \times 48$ & $70 \mathrm{x} 46$ & $64 \mathrm{x} 44$ \\
\hline 7 & Fabric count $($ warp/inch $\mathrm{x}$ weft/inch) & $31.5+13.2$ & $20.6+17.0$ & $22.6+18.2$ \\
\hline 8 & $\begin{array}{l}\text { Fabric cover factor* }(\mathrm{K})=\mathrm{k} 1 \text { (warp) } \\
+\mathrm{k} 2 \text { (weft) }\end{array}$ & & \multicolumn{2}{|c|}{} \\
\hline
\end{tabular}

*Cover factor is defined as the area of a fabric covered by a set of threads

Number in brackets indicates standard deviation

\section{Investigation of colour change}

Figure 2 is an array of digital images showing the full matrix of denim fabric weights, laser power and grayscale levels. It is evident from these images that 0 to $20 \%$ grayscale already provides sufficient visual contrast for effective half tone images. 


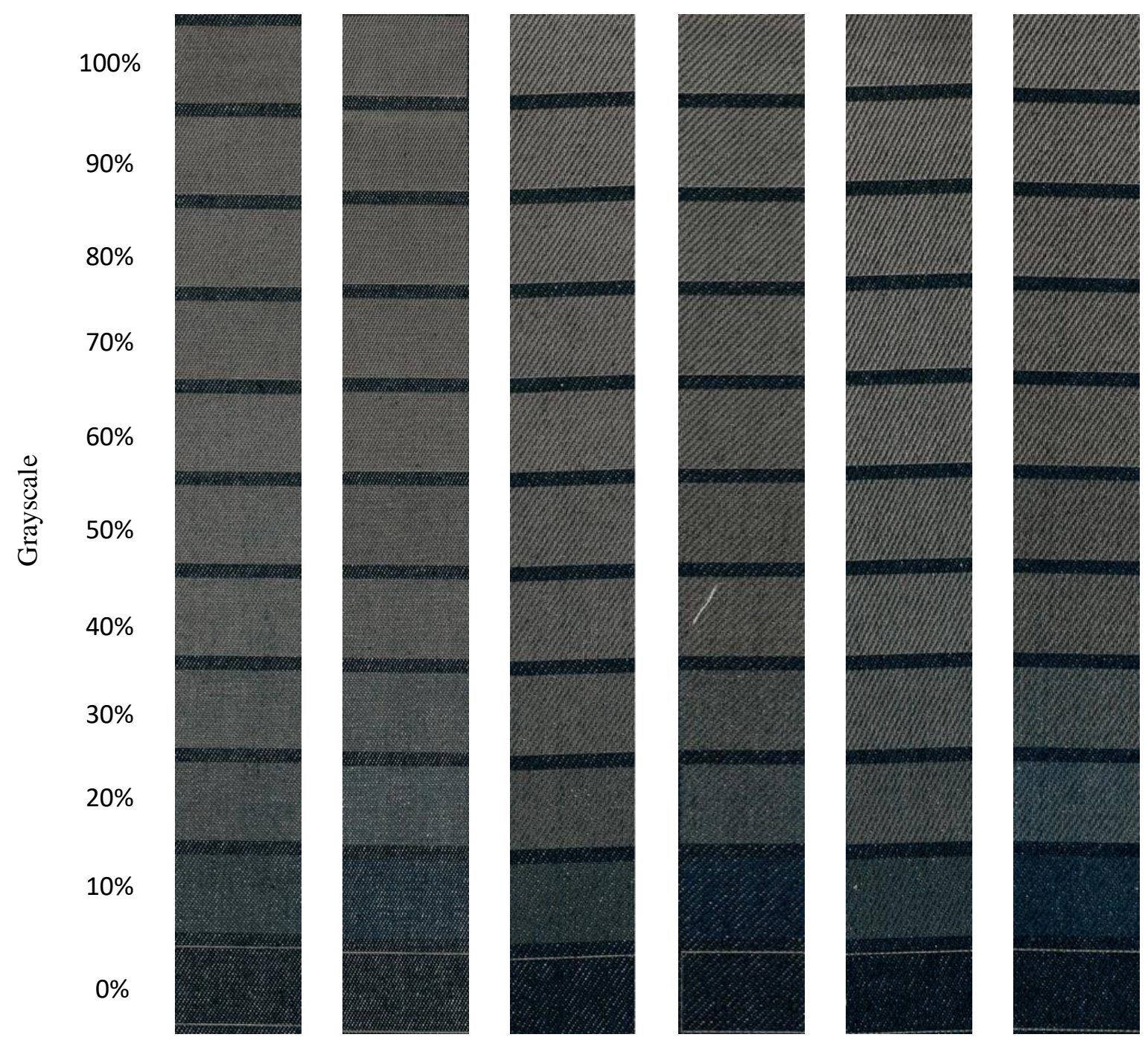

LW 50\% LP LW 100\% LP MW 50\% LP MW 100\% LP HW 50\% LP HW 100\% LP

Figure 2. Sample laser etched denim fabrics (laser irradiation in weft direction)

It could be observed (Figure 3) that colour brightness was affected by laser power in all the three-different type of fabric weights, in the case of light weight fabric, the brightness of colour is marginally low for $100 \%$ laser power compared to $50 \%$ laser power. This could be due to the high laser power that had removed colour from the fabric surface compared to 50\% laser power. This trend was similar for MW and HW fabrics, where the fabrics treated at 50\% power had slightly higher colour brightness across all the grayscale ratings compared to those treated at $100 \%$ power. Figure 4 illustrates colour hue for various fabric weight at 50\% and $100 \%$ laser power. As the grayscale increases colour hue changes from blue shade to greenish yellow and this trend was similar across all fabric weights. Yellowing become noticeable at higher grayscale due to oxidation of cellulose fibres across all fabrics. 
Table 2. Physical parameters of Fabrics

\begin{tabular}{|c|c|c|c|c|c|}
\hline S.No & \multicolumn{2}{|c|}{ Fabric parameters } & LW & MW & HW \\
\hline \multirow{4}{*}{1} & \multirow{4}{*}{$\begin{array}{l}\text { Fabric tensile } \\
\text { strength }(\mathrm{N}) \\
\text { (gauge length } 200 \mathrm{~mm} \text { ) }\end{array}$} & Warp direction & $\begin{array}{l}559.5 \\
( \pm 5.6)\end{array}$ & $\begin{array}{c}850.2 \\
( \pm 34.5)\end{array}$ & $\begin{array}{l}1175.2 \\
( \pm 45.6)\end{array}$ \\
\hline & & Weft direction & $\begin{array}{r}592.5 \\
( \pm 22.0)\end{array}$ & $\begin{array}{r}623.6 \\
( \pm 15.9)\end{array}$ & $\begin{array}{r}777.0 \\
( \pm 16.1)\end{array}$ \\
\hline & & $\begin{array}{c}\text { Elongation in warp } \\
\text { direction }(\%)\end{array}$ & $\begin{array}{c}36.2 \\
( \pm 0.50)\end{array}$ & $\begin{array}{c}38.8 \\
( \pm 3.9)\end{array}$ & $\begin{array}{c}43.0 \\
( \pm 1.4)\end{array}$ \\
\hline & & $\begin{array}{c}\text { Elongation in weft } \\
\text { direction }(\%)\end{array}$ & $\begin{array}{c}13.6 \\
( \pm 0.05)\end{array}$ & $\begin{array}{c}13.7 \\
( \pm 0.14)\end{array}$ & $\begin{array}{c}13.2 \\
( \pm 0.61)\end{array}$ \\
\hline 2 & Air permeability (cc & & $\begin{array}{c}65.0 \\
( \pm 0.0)\end{array}$ & $\begin{array}{c}47.0 \\
( \pm 1.7)\end{array}$ & $\begin{array}{c}53.0 \\
( \pm 2.9)\end{array}$ \\
\hline & $\begin{array}{c}\text { Colourfastness to } \\
\text { rubbing }\end{array}$ & Warp wet & 1 & 1 & $1 / 2$ \\
\hline & (greyscale rating - & Warp dry & 4 & $3 / 4$ & $4 / 5$ \\
\hline 3 & 5- no perceived & Weft wet & $1 / 2$ & $1 / 2$ & $1 / 2$ \\
\hline & $\begin{array}{l}\text { 1- high colour } \\
\text { change }\end{array}$ & Weft dry & 4 & $3 / 4$ & $4 / 5$ \\
\hline
\end{tabular}

Figure 5 indicates saturation values between 2 to $10 \%$, these relatively low values are due to the make-up of denim which is woven with blue colour (warp) and white colour (weft) yarns. For LW fabric, colour saturation for 50\% laser power was lower until $30 \%$ grayscale where removal of indigo dyes was less compared to $100 \%$ LP. Saturation trends were similar for MW and HW fabrics where higher values were obtained for 10-40\% grayscale; colour saturation decreased to $50-80 \%$ grayscale and sudden increase in saturation at $90-100 \%$ grayscale. 


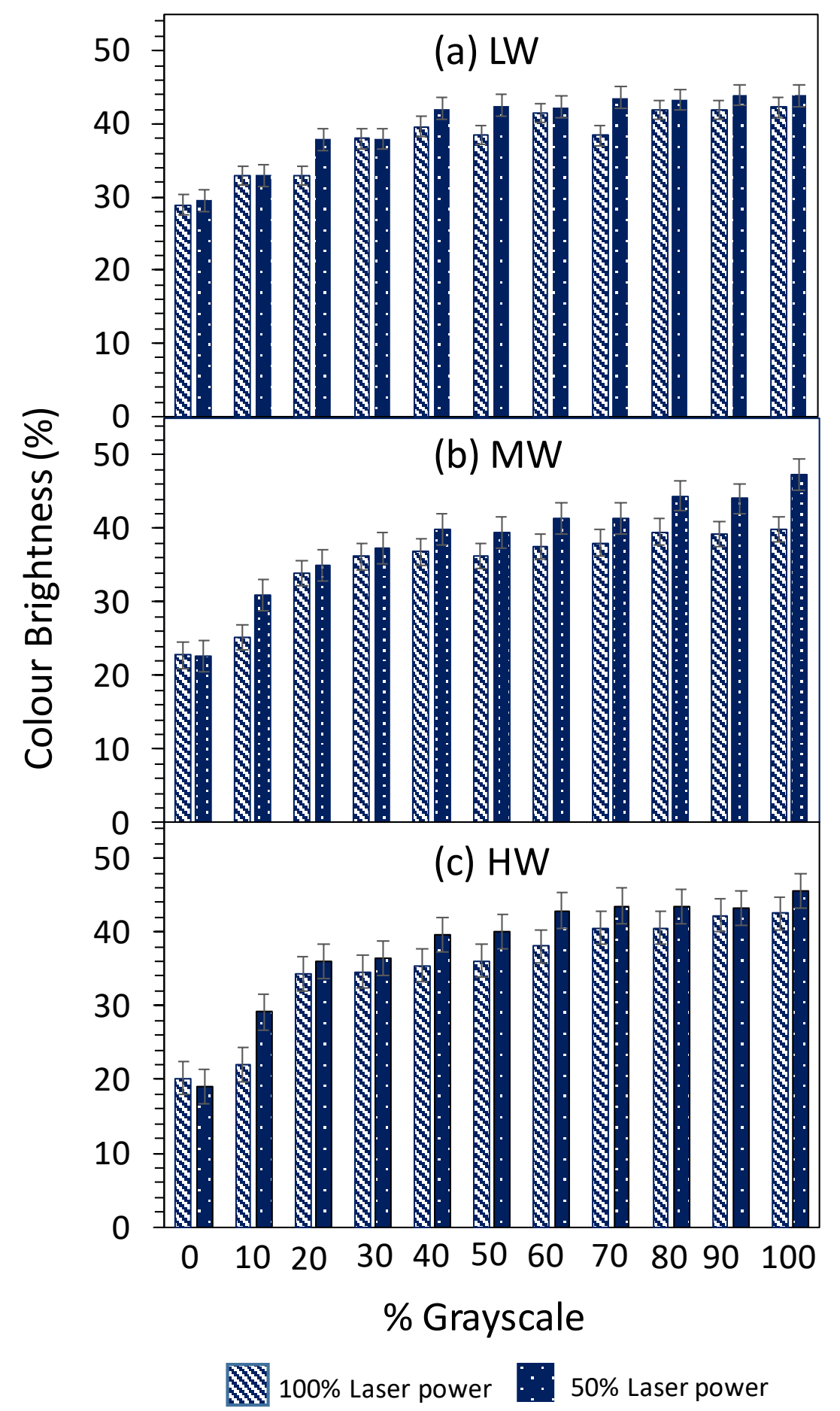

Figure 3 Colour brightness for different grayscale rating and laser power, (a) light weight (LW), (b) medium weight (MW)and (c) heavy weight fabrics (HW). 


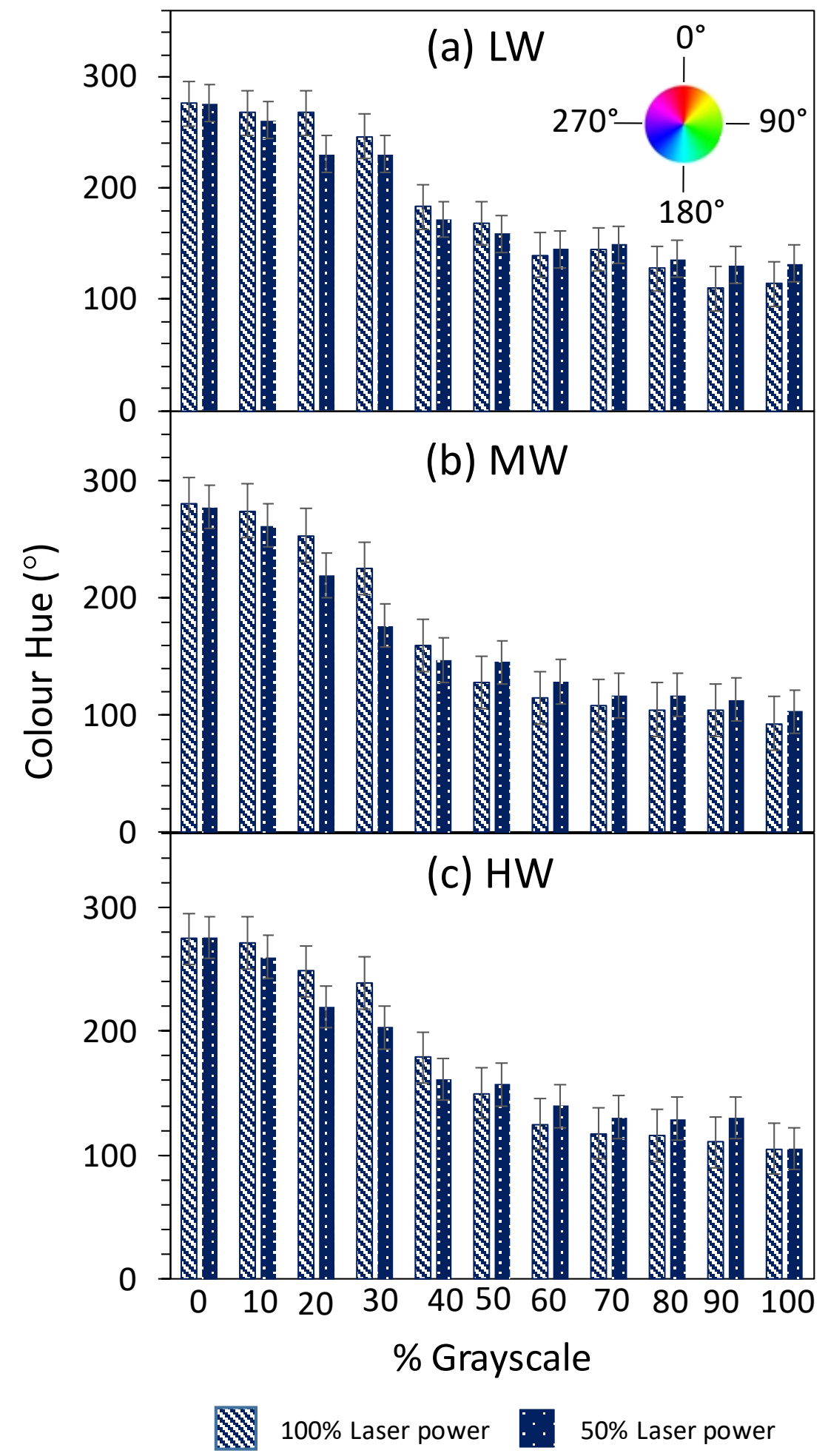

Figure 4 Colour Hue $(\mathrm{H})$ for various fabric weight and laser power; (a) light weight, (b) medium weight and (c) heavy weight fabrics. Note for convenience, the colour hue scale is reproduced on (a). 


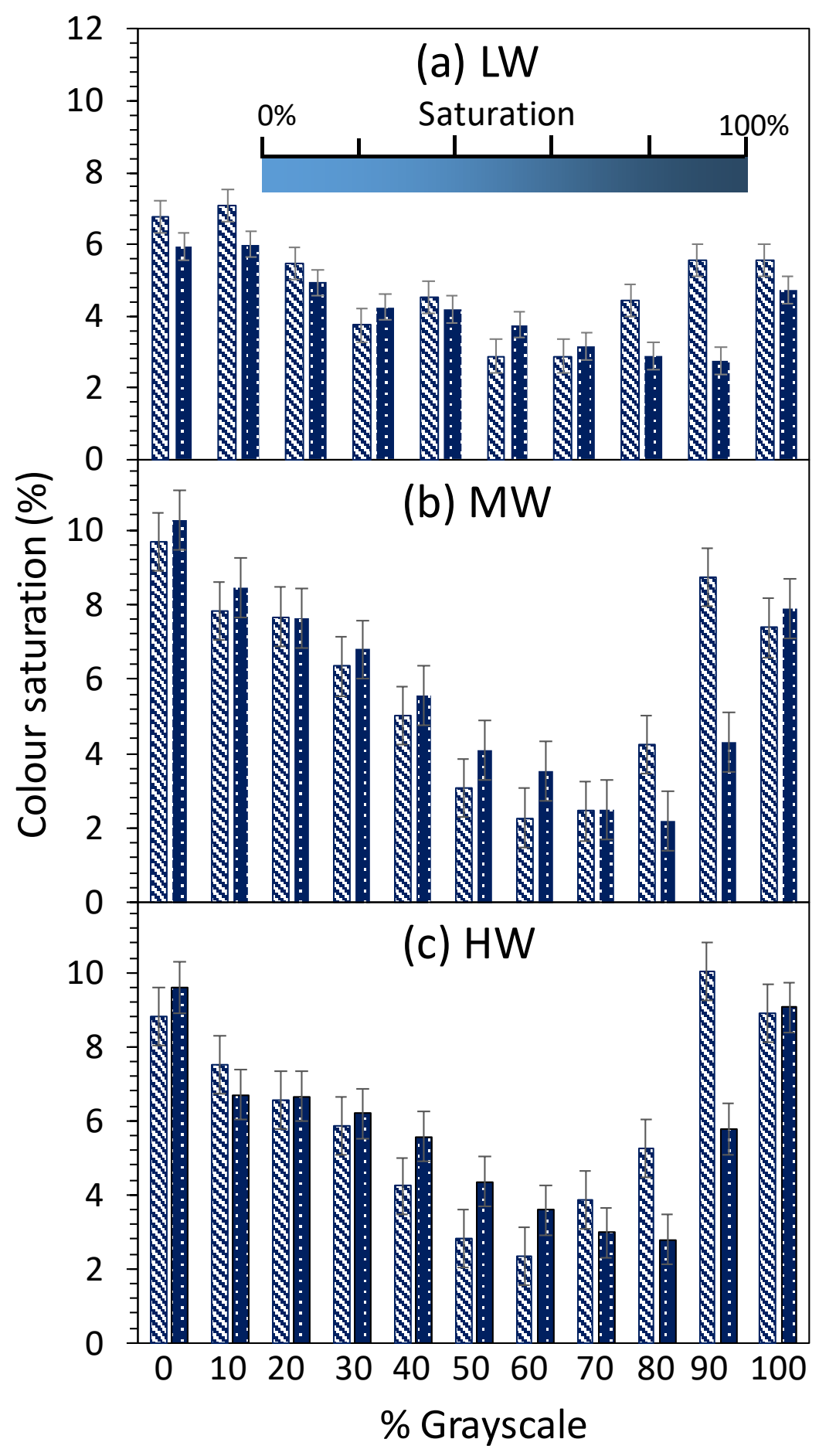

100\% Laser power

50\% Laser power

Figure 5. Colour saturation for various fabric weight and laser power, (a) light weight, (b) medium weight and (c) heavy weight fabrics. Note, a schematic showing the saturation scale is included in (a). 
(a) $50 \%$ Laser power

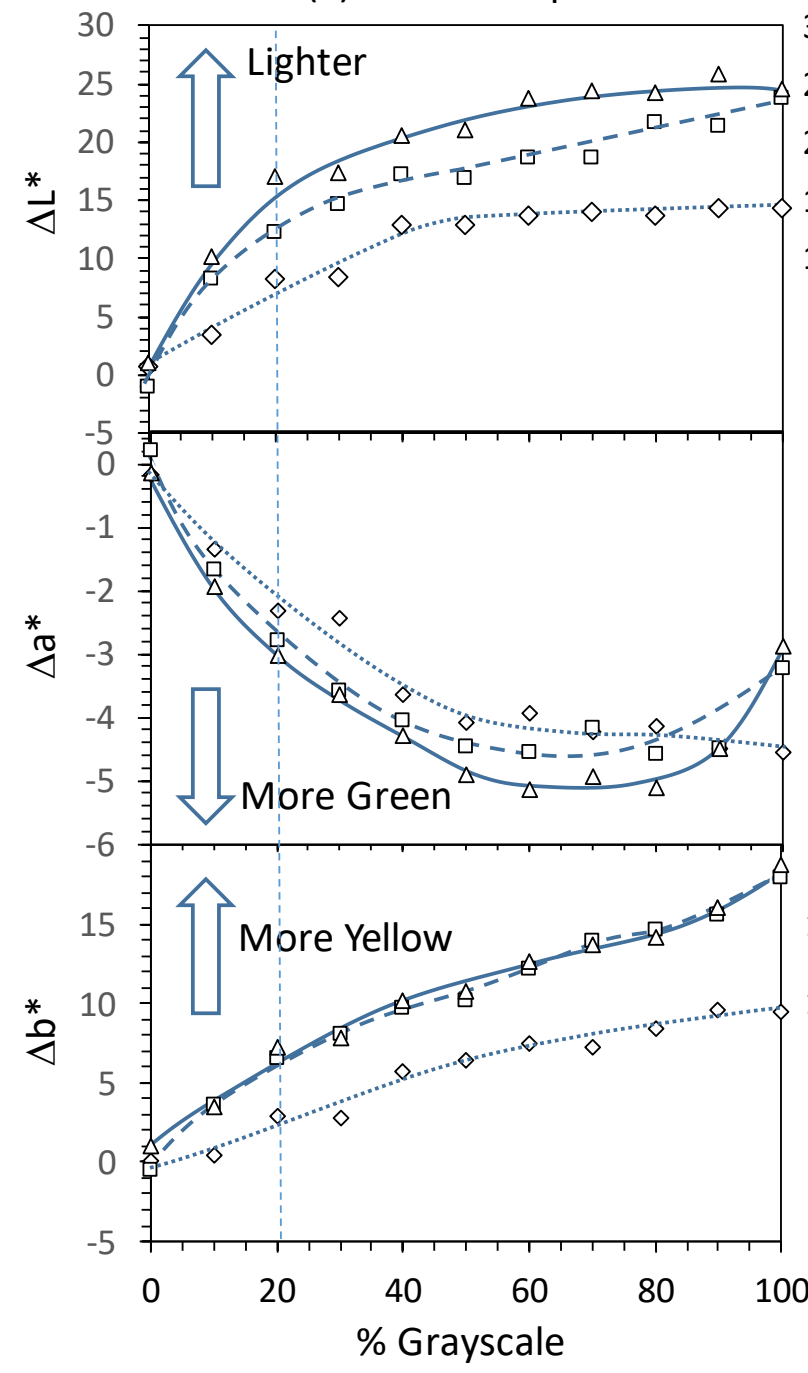

(b) $100 \%$ Laser power

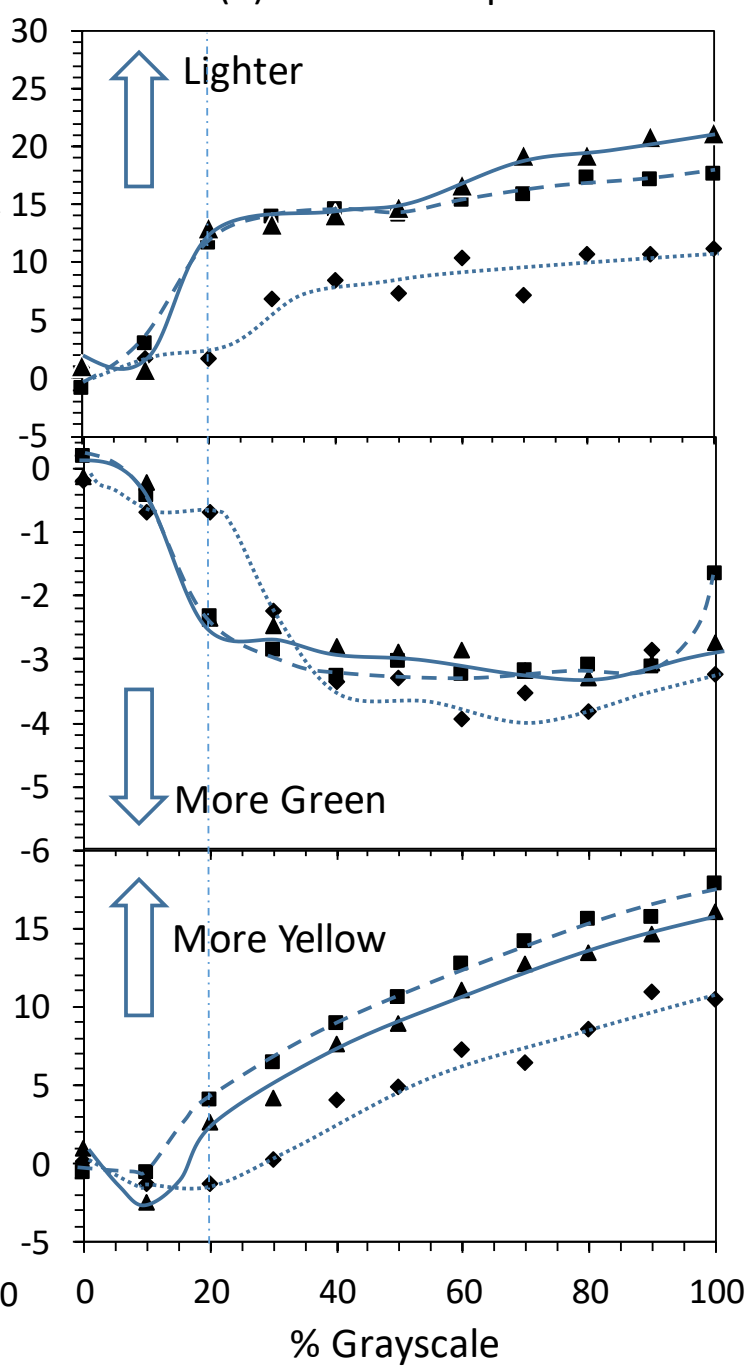

Figure 6. $\Delta \mathrm{L}^{*}, \Delta \mathrm{a}^{*}$ and $\Delta \mathrm{b}^{*}$ data plotted as a function of $\%$ grayscale for (a) $50 \%$ laser power and (b) $100 \%$ laser power. $\diamond \mathrm{LW}, \square \mathrm{MW}, \triangle \mathrm{HW}$, open symbols $50 \%$ laser power, solid symbols $100 \%$ laser power.

Figure 6 gives the colour change analysis $\Delta \mathrm{L}^{*}, \Delta \mathrm{a}^{*}, \Delta \mathrm{b}^{*}$ for $50 \%$ laser power and $100 \%$ laser power respectively across three types of fabrics. The $\Delta \mathrm{L}^{*}$ versus grayscale plot for $50 \%$ laser power indicates more rapid increase in brightness or lighter in shade between 0 and 20\% grayscale. This was also evident in Figure 3, where colour brightness was higher for 50\% laser power compared to $100 \%$ laser power for all fabrics. At $100 \%$ laser power, $10 \%$ grayscale did not remove much colour in the MW and HW fabric, though a step increase in $\Delta \mathrm{L}^{*}$ was observed at $20 \%$ grayscale (also refer Figure 2). The $\Delta \mathrm{L}^{*}$ values observed at high grayscale were lower at $100 \%$ laser power due to charring effects. These measurements supported by direct observation; $50 \%$ laser power provided improved contrast due to reduced charring effects and 
most of the visually observed change from blue to a whiter shade occurred between 0 and 20\% grayscale. The downward $\Delta \mathrm{a}^{*}$ trend, observed in all cases, shows that the samples are moving toward green tint, this is because the remaining indigo blue dye is combining with yellow tints arising from oxidation of the cellulose. At $50 \%$ laser power, the reduction in $\Delta \mathrm{a}^{*}$ is greater than at $100 \%$ laser power due to reduced charring effects relative to those observed at the higher laser power. The intensity of the yellow tint $\Delta \mathrm{b}^{*}$ increases steadily with increasing grayscale, though at lower grayscale (0 to 20-30\%) the amount of yellowing is barely perceptible to the eye. Interestingly, the LW fabric shows a noticeably different $\Delta \mathrm{L}^{*}$ and $\Delta \mathrm{b}^{*}$ trend with these parameters changing more slowly and reaching lower values at $100 \%$ grayscale compared to MW and HW fabrics. This may be due to the initial colour of the LW fabric being lighter and therefore not able to absorb so much energy from the laser light.

Reflectance curves from the spectrophotometer gives the depth of the shade of the material in the visible spectrum (Figure 7). Generally, the reflectance values increased when the grayscale increased from 10 to $100 \%$, which meant that the shade of denim fabric is pale compared to unetched denim fabric. This is due to removal of dyes from the fabric. In the case of $100 \%$ laser power (Figure 7 (a)), for all the three fabric weights LW, the curve with dotted lines and cross (for unetched standard fabric) indicates the low reflectance levels compared to 10 to $100 \%$ grayscale laser etching. In the case of LW fabric, peaks were observed at 400 to $420 \mathrm{~nm}$, 580-600 and $700 \mathrm{~nm}$. This trend was similar in the case of other fabric weights and is line with previous findings (Kan, 2014a). The higher reflectance values indicate depth of the shade will be pale and vice versa. In the case of 50\% laser power (Figure 7(b)), peaks were observed for LW fabric in the range $520-540 \mathrm{~nm} ; 700 \mathrm{~nm}$; for MW and HW peaks were observed in the range $580-600$ and $700 \mathrm{~nm}$. In the case of 50\% laser power, the reflectance curves for $10 \%$ to $100 \%$ grayscale shades were different to the curve for unetched fabrics (LW, MW and HW). For LW fabric, peaks were observed in the range 520-560 nm; and $700 \mathrm{~nm}$; whilst for MW fabric 560600 and $700 \mathrm{~nm}$ and HW 520-600 and $700 \mathrm{~nm}$. The overall shape of reflectance curves for 50\% and $100 \%$ laser power was similar, when the reflectance values are higher the depth of the shade is pale.

The Kubelka-Munk function provides the colour yield of fabrics. The K/S values for pristine denim fabric was distinctly different to laser treated samples -10 to $100 \%$ grayscale. This can be observed from Figure 8, for instance in the case of $100 \%$ laser treatment, the K/S curves for untreated fabric (dotted curve with cross for MW fabric) was different to 20 to $100 \%$ grayscale. This also indicated that as the laser treatment is increased, the K/S values were lower compared to untreated fabric, indicating colour fading due to removal of indigo dyes from denim fabric surface. This trend was also noticed for $50 \%$ laser power treated samples and similar to LW and HW fabric. 


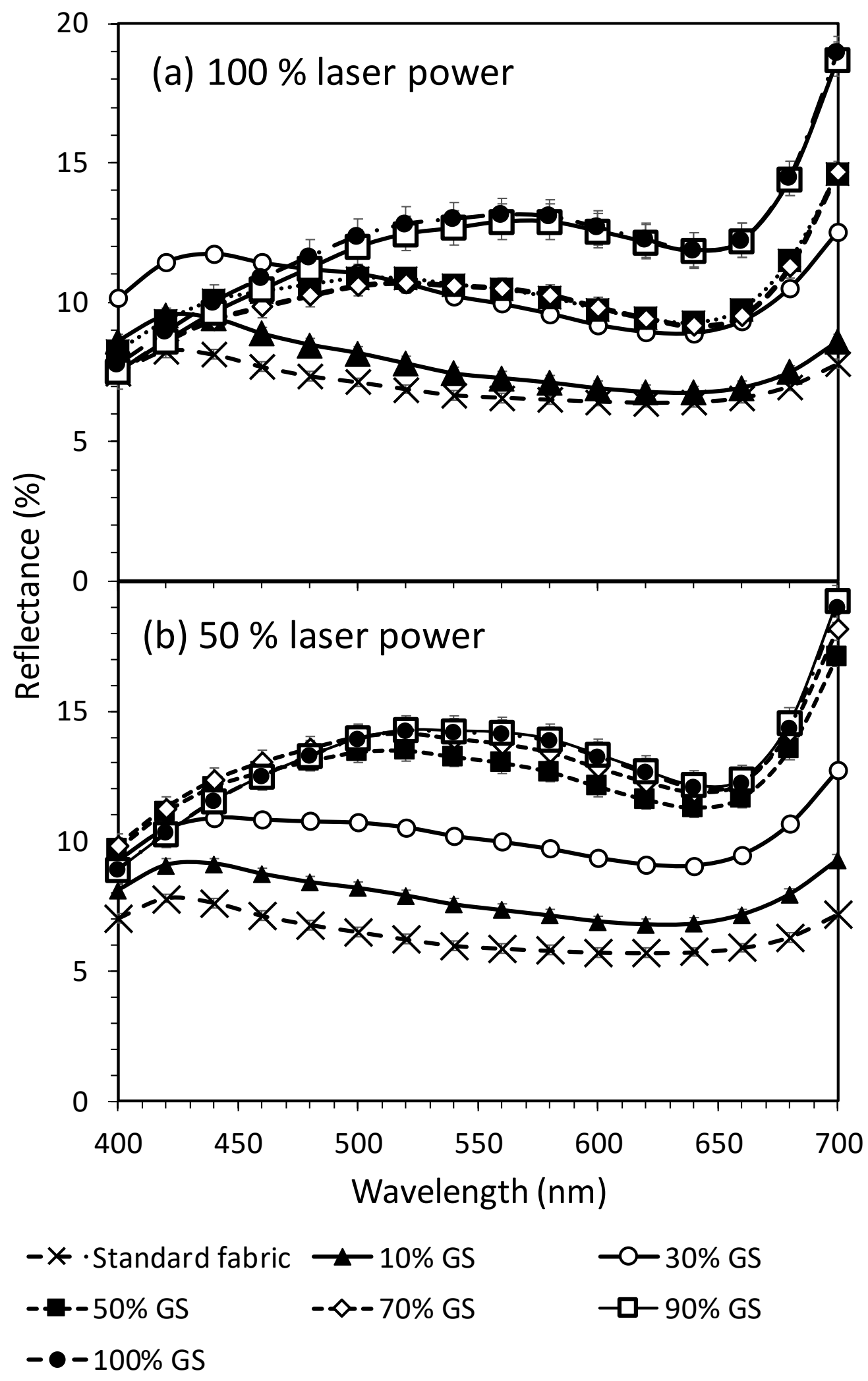

Figure 7. Spectrophotometer - reflectance curves at (a) $100 \%$ laser power and (b) $50 \%$ laser power for 0 to $100 \%$ grayscale (GS) LW fabric 

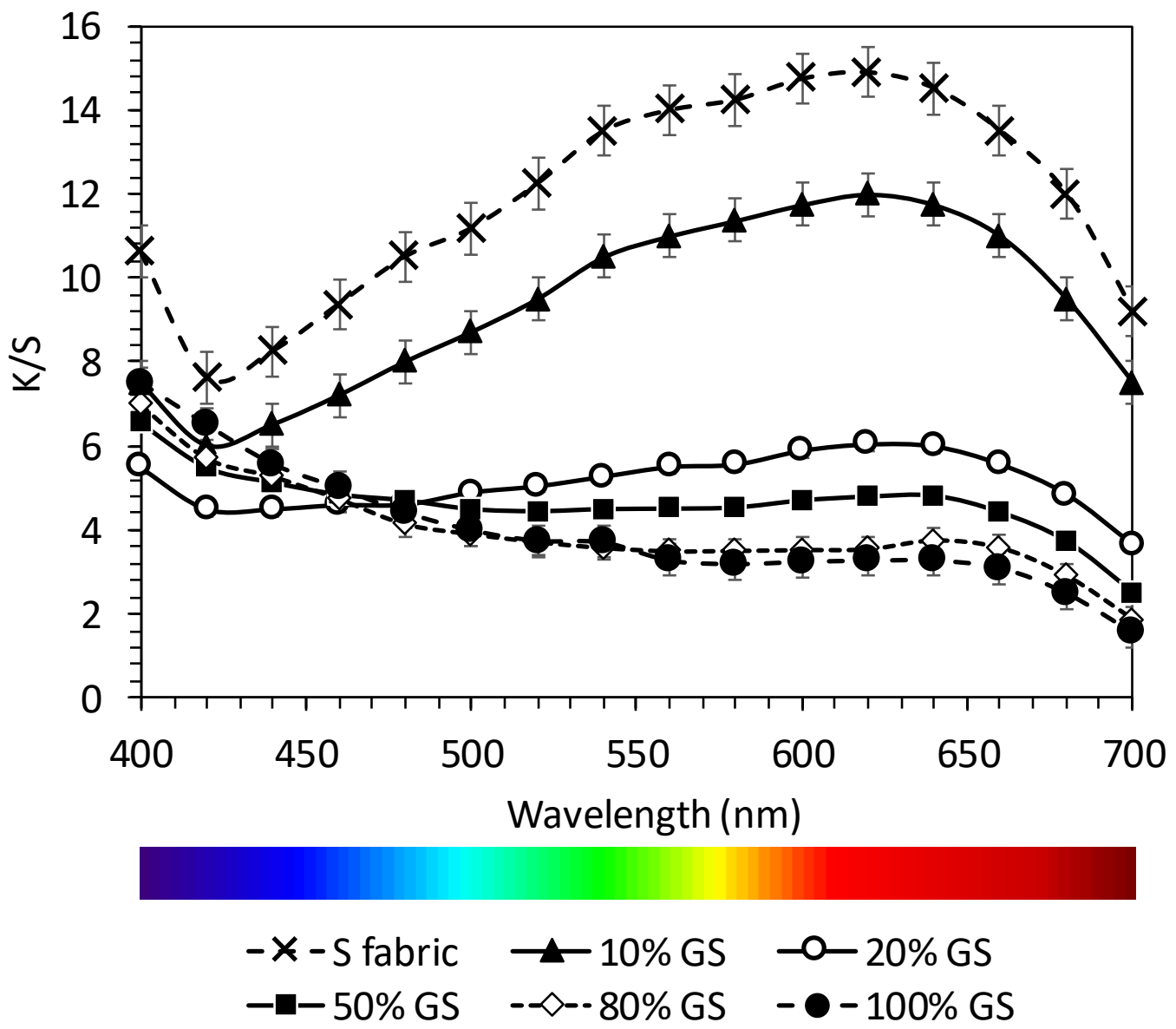

Figure 8. Spectrophotometer -colour yield (K/S value) at $100 \%$ laser power for 0 to $100 \%$ grayscale - MW fabric

\section{Fabric Morphological Analysis}

It can be noted that SEM pictures were focused on laser effect on warp yarns in the fabric, as the warp yarns float (indigo dyed) in a denim fabric. The control fabric ( $0 \%$ laser treatment) was compared across various grayscale treatment under three magnification scales, 400x and $5 \mathrm{Kx}$. As the grayscale increases, the surface damage to the fabric increases. It can be observed that the fabric structure was unaffected for all fabrics in the case of the 50\% laser treatment. However, in the case of $100 \%$ laser treated samples $100 \%$ to $80 \%$ grayscale surface distortion was prominent, the fabrics collapsed which meant the fabric was damaged completely due to laser etching. The laser beam etching was consistent such that burning effect on the material occurred on the warp yarns and this can be observed at 10-20\% grayscale (400x magnification) in Figure 9. From the SEM images of the degraded regions it appears that cellular structures have been formed in line with those observed by Jucienè et al., (2014). 
Medium Weight Denim fabric - Scanning Electron Microscope surface analysis

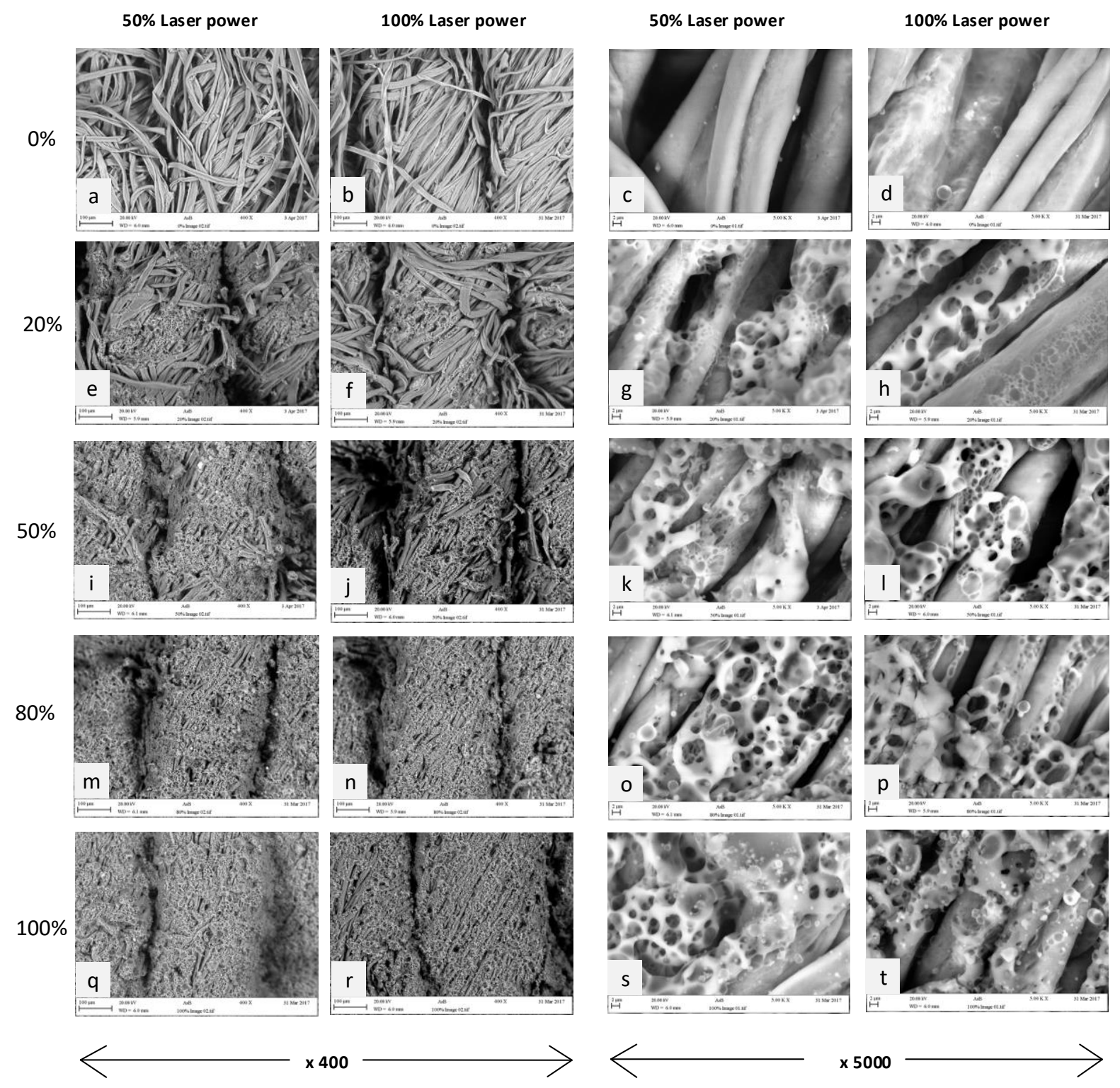

Figure 9. Scanning Electron Microscope analysis medium weight denim fabric

The cellular structures form as a result of rapid heating and pyrolysis into volatile fractions that then give rise to the pores in the remaining material. The latter effect is more noticeable at $100 \%$ laser power.

It could be noted from the graph (Figure 10) that the laser treatment affected fabric surface thickness from $0 \%$ to $100 \%$ grayscale rating. There is a gradual and consistent decrease in thickness of the denim fabric as the laser treatment varies from 0 to $100 \%$. 


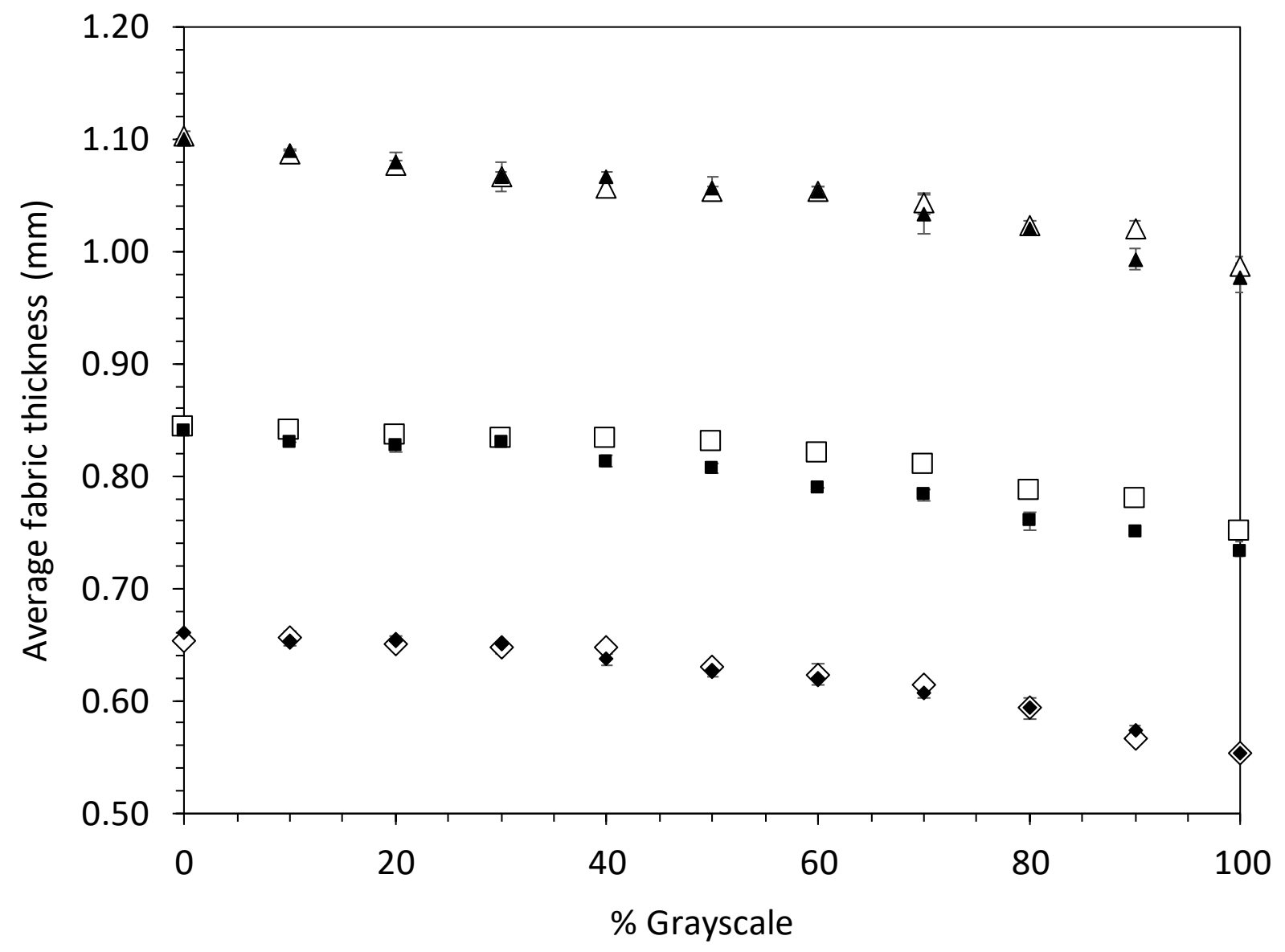

Figure 10. Fabric thickness variations for various fabric weight and $100 \%$ and $50 \%$ laser power treatment. $\diamond \mathrm{LW}, \square \mathrm{MW}, \triangle \mathrm{HW}$, open symbols $50 \%$ laser power, solid symbols $100 \%$ laser power.

This indicated that as the laser engraving increases, the fabric loses its surface fibres resulting in difference in fabric thickness, as observed in SEM images. At 20\% grayscale most of the indigo dye is removed from the exposed fibres on the fabric surface compared to $0 \%$ grayscale. As the dye itself contributes insignificantly to thickness, it is not surprising that the reduction in thickness at $20 \%$ grayscale is largely insignificant. At $60 \%$ grayscale a slight yellow tint becomes visually noticeable and the reduction in thickness becomes more significant, indicating degradation and volatilisation of the cellulose polymer. Interestingly there is a marginal difference between $50 \%$ and $100 \%$ laser power in each fabric type. This may be explained by the increased generation of cellular structures at $100 \%$ laser power; there is less material present, but the structure is less dense and occupies similar volume. The differences in thickness between $100 \%$ grayscale and $0 \%$ grayscale are similar indicating similar levels of material removal, regardless of initial fabric thickness. 


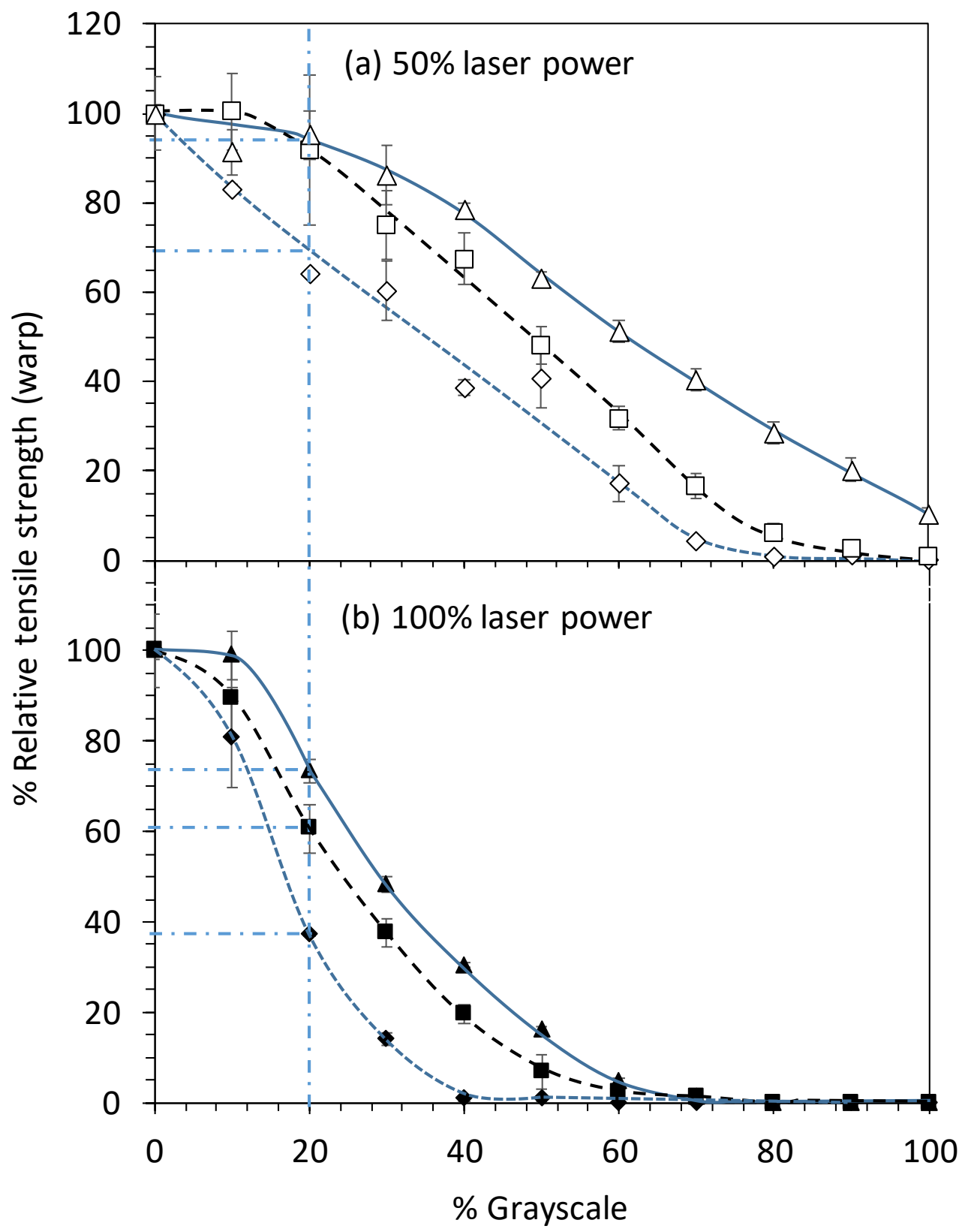

Figure 11. Relative breaking load (in the warp direction) versus grayscale; (a) $50 \%$ laser power and (b) $100 \%$ laser power. $\diamond \mathrm{LW}, \mathrm{O} \mathrm{MW}, \triangle \mathrm{HW}$, open symbols $50 \%$ laser power, solid symbols $100 \%$ laser power.

Percentage relative tensile strength (warp direction) is plotted against grayscale in Figure 11. Use of relative strength enables the effect of increasing laser damage to be highlighted without interference from the expected effect of fabric thickness on tensile strength. Note the strength values for the pristine fabrics are provided in Table 2. The amount of material removed / degraded in relation to the initial thickness will of course be expected to have a significant effect and is apparent in the data obtained. The strength of the LW fabric falls to zero at relatively the lowest grayscale, followed respectively, by the MW and HW fabrics. If $0 \%$ to 
$20 \%$ grayscale is considered the maximum range for obtaining patterns with good contrast, then it is evident that at 50\% laser power (Figure 11(a)), the HW and MW fabrics are barely reduced in strength at $20 \%$ grayscale. However, at the latter grayscale, the strength of the LW fabric fell to approximately $68 \%$ of its initial value. This indicates that great care must be taken with laser patterning of LW denims. At 100\% laser power (Figure 11(b)), the strengths of the HW, MW and LW fabric fell, respectively, to approximately $74 \%, 62 \%$ and $37 \%$ of their original values. This observation indicates that laser power must be carefully optimised if fabric strength is to be retained. The reduction in strength could be attributed to loss of surface fibres and yarns contributing toward the fabric strength. Increasing laser intensity causes damage to the cellulose fibre on the surface of the fabric and this depends on laser processing parameters and fabric thickness and fabric density. Bosman (2007) highlighted that laser beam produces a thermal effect on denim fabric that causes the indigo dye to sublime, revealing the undyed yarn, and oxidises the surface of the textile fibre. For the laser treated fabrics, strength falls away very rapidly with decreasing thickness, this may be due to the formation of cellular structures of lower density and hence minimal load bearing capacity. The amount of degradation occuring before onset of formation of cellular structures may also be sufficient to noticeably reduce strength.

Air permeability demonstrates the rate of airflow perpendicularly through the fabric and it affects the comfort. In the pristine samples, the LW denim has predicticably higher air permeability than the MW and HW samples (Figure 12). This could be attributed to the pores within the fabric structure allowing free air flow. The cover factor is also related to the formation of pores within the fabric structure. Cover factor for LW fabric in the weft direction was lower compared to MW and HW fabric. At 50\% laser power and 10\% grayscale (Figure 12(a)), the permeability of the LW sample reduced to the same level as the other samples in their pristine state. All samples showed little variation until 60 to $70 \%$ grayscale where a reduction in permeability, followed by a gradual increase from 70 to $100 \%$ grayscale was observed in the LW and MW fabrics. The HW fabric showed no such increase, infact the latter material showed a slow but generally steady decrease in permeability over the whole grayscale range. The SEM images show formation of cellular structures resulting from degradation giving volatile products combined with localised melting and solidification and possible fusion of fibres. The latter, combined with generation of predominantly closed cell foam-like structures, is likely to reduce permeablity. However, if subtantial material is volatilised and the foam-like structures become more open cell in nature, then permeablity could increase. The latter, possibly combined with overall removal of material may explain the increasing permeability trends observed for the LW and MW fabrics at $100 \%$ laser power (Figure 12(b)) especially between $80-100 \%$ grayscale. This could also be due to the fact that laser engraving produced 
open cellular structures as seen from SEM images that resulted in sudden increase in air flow (see table 1). The permeability of the HW fabric did not change significantly over the entire grayscale range, this may be due to dominance of the undegraded material making up the total thickness.

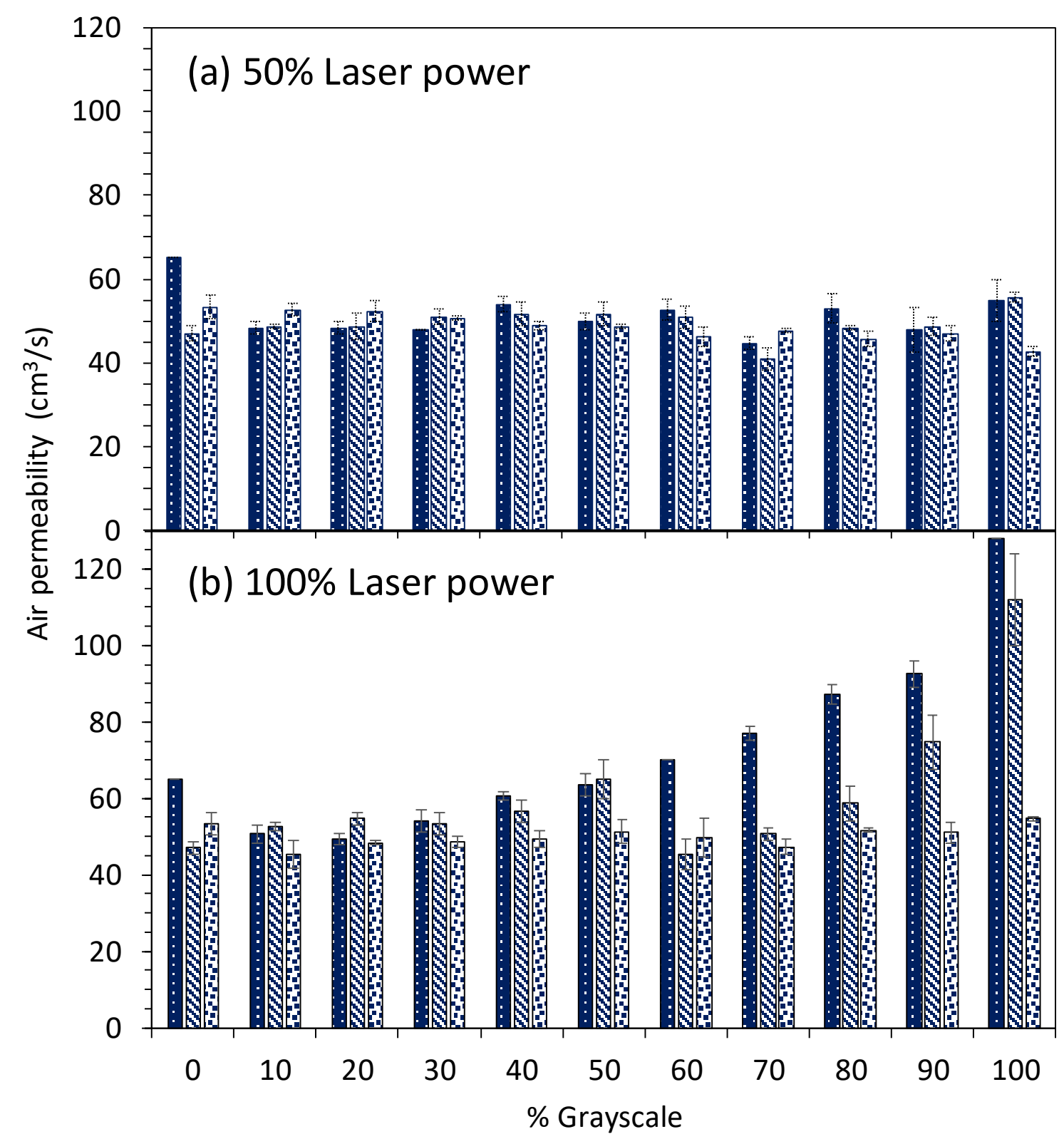

ㄴ. $\operatorname{LW}(100 \% \mathrm{LP}) \quad$ MW $(100 \% \mathrm{LP})$ 固 HW (100\% LP)

Figure 12. Air permeability of the fabrics at (a) 50\% Laser Power and (b) $100 \%$ Laser power. 


\section{ATR-FTIR}

The IR spectrum of cellulose has been studied in some detail over the last decades. A number of studies include correlation tables for the main absorption bands (Gilbert 1993 and Chung, 2004). All the fabric samples were examined and it became apparent that the trends related to the effect of grayscale level on cellulose structure within each set (based on fabric weight and laser power) were the same. This is due to the micron-scale penetration depth of the evanescent wave emerging from the internal reflection element.

Figure 13 shows the $\mathrm{OH}$ and $\mathrm{CH}$ stretching region of the spectra obtained for the light weight, $100 \%$ laser power series. The most immediately obvious effect of the laser engraving (at grayscales between 10 and 50\%) is broadening and smoothing of the $\mathrm{OH}$ stretching bands; increasing grayscale to $100 \%$ did not have any significant effect on this region of the IR spectrum. The $\mathrm{OH}$ stretching region and the effect of thermal degradation on the latter has been studied using a chemometric approach (Kokot et al, 2002); the changes observed on laser engraving are also similar to those observed by Kokot et al in their thermal degradation study. In the pristine and $10 \%$ grayscale samples, there were well-defined $\mathrm{H}$-bonded $\mathrm{OH}$ stretching peaks (at 3400, 3360 and $3267 \mathrm{~cm}^{-1}$ ). The latter two were due to intra-chain hydrogen bonding and inter-chain hydrogen bonding, respectively (Kokot et al., 2002). Reduction in vibration intensity of these specific interchain hydrogen bonds is certainly consistent with destruction of the crystal structures and reduction in the specific intra-chain hydrogen bonds may well be related to destruction of the cellulose chains themselves. Heat build-up in the fibres and the resulting degradation has wrecked the relative uniformity of the $\mathrm{OH}---\mathrm{H}$ bond distances within the crystal structures, leading to broader range of $\mathrm{OH}---\mathrm{H}$ bond distances and therefore a wider distribution of vibration frequences, hence broader, less intense absorption peaks. There has also been a slight shift on the $\mathrm{OH}$ band envelope to higher vibration frequency this is consistent with less interaction between $\mathrm{OH}$ groups, an effect that further supports a reduction in the overall level of structural order. Other investigators such as Ferrero et al., (2002) and Hung et $a l .$, (2017) have also observed broadening / merging of the $\mathrm{OH}$ stretching bands as a result of thermal / electron beam and $\mathrm{CO}_{2}$ laser degradation of linen and cotton fabrics.

The other important absorption bands are the $\mathrm{C}-\mathrm{H}$ stretching bands. The asymmetric methylene $\mathrm{C}-\mathrm{H}$ stretching region is particularly interesting as in the pristine sample there is a weak sharp peak at $2915 \mathrm{~cm}^{-1}$ which is consistent with self-assembled long alkyl chains possibly associated with a wax, either natural and surviving the dying process, picked up from processing machinery, or added as a finish / process aid. At 10\% grayscale this peak is reduced in intensity indicating possible removal of a fraction of this waxy material. Between 10 and $50 \%$ grayscale the methylene C-H asymmetric stretching frequency shifts slightly to $2920 \mathrm{~cm}^{-1}$, this indicates 
that a fraction of the alkyl tails are in liquid-like state of dissorder that is consistent with oxidation.

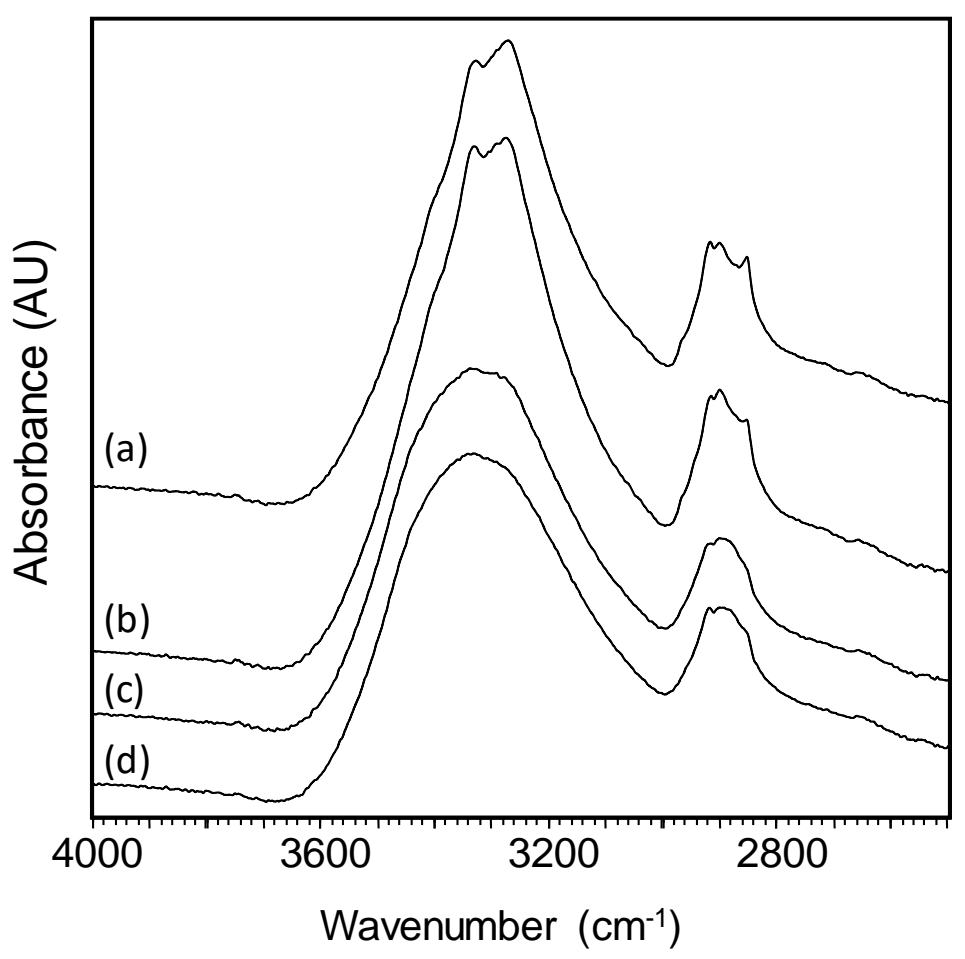

Figure 13. Stacked ATR-FTIR spectra showing $\mathrm{OH}$ and $\mathrm{CH}$ stretching regions for the light weight denim 100\% laser power series; (a) pristine denim, (b) $10 \%$ grayscale, (c) 50\% grayscale and (d) $100 \%$ Grayscale.

Stacked spectra showing the region from 1800 to $450 \mathrm{~cm}^{-1}$ are shown in Figure 14. The main absorptions are assigned in Table 3. It is immediately evident that absorptions associated with the indigo dye were superimposed on the spectrum for cotton (cellulose). As grayscale increases, the intensity of the indigo related bands decreases, however some indigo bands remain even at $100 \%$ grayscale. Progressively severe damage to the cellulose polymer can also be seen in the spectra as grayscale increases. The absorptions from 1140 to $920 \mathrm{~cm}^{-1}$ (Table 3) become broader and hence much less defined such that the peaks tended to merge together. In addition to the latter the in-phase ring stretching band at $900 \mathrm{~cm}^{-1}$ becomes more intense as such movement is less confined by crystalline order. These observations are consistent with destruction of the crystalline order in the cellulose polymer and chain scission. Considering the level of damage seen in the SEM images and the FTIR spectra, the amount of non-volatile carbonyl compounds formed because of the laser engraving process is not particularly significant; there is only a relatively low level of carbonyl species observed (broad peak, centred at about $\left.1710 \mathrm{~cm}^{-1}\right)$. 
Table 3. Assignments for the key cotton and indigo absorptions in pristine denim (functional group and fingerprint region only) (Cotton assignments: Gilbert, 1993 and Chung, 2004. Indigo assignments, Baran et al., 2010)

\begin{tabular}{|c|c|}
\hline \multicolumn{2}{|r|}{ Table 3 ATR - FTIR Absorbance spectra values } \\
\hline $\begin{array}{c}\text { Peak position } \\
\left(\mathrm{cm}^{-1}\right)\end{array}$ & Assignment \\
\hline Ca. 1740 & Carbonyl stretch - esters, fatty acids etc. \\
\hline $\begin{array}{l}\text { Centred at } \\
1640 \text { broad } \\
\text { underlying }\end{array}$ & $\begin{array}{l}\text { Cotton - H-OH bending due to adventitious surface water and also water } \\
\text { within the cellulose structure }\end{array}$ \\
\hline 1629 & Indigo - carbonyl stretching \\
\hline $1612-1609$ & Indigo $-\mathrm{C}-\mathrm{C}$ in plane stretching in 6 membered ring \\
\hline 1584 & Indigo - As above \\
\hline 1480 & $\begin{array}{l}\text { Indigo }- \text { symmetric in phase } \mathrm{C}-\mathrm{H} \text { rocking and } \mathrm{C}-\mathrm{C} \text { stretching in } 6 \\
\text { membered ring }\end{array}$ \\
\hline 1460 & $\begin{array}{l}\text { Indigo }- \text { asymmetric out of phase } \mathrm{C}-\mathrm{H} \text { rocking and } \mathrm{C}-\mathrm{C} \text { stretching in } 6 \\
\text { membered ring }\end{array}$ \\
\hline 1389 & Indigo $-\mathrm{N}-\mathrm{H}$ rocking and $\mathrm{C}-\mathrm{N}$ stretching \\
\hline $\begin{array}{c}1370 \text { and } \\
13201300\end{array}$ & Cotton - Symmetric C-H bending C-H \\
\hline 1315 & $\begin{array}{l}\text { Indigo }-\mathrm{C}-\mathrm{C} \text { stretching in } 6 \text { and } 5 \text { membered ring and out of phase } \\
\text { asymmetric } \mathrm{C}-\mathrm{H} \text { rocking }\end{array}$ \\
\hline 1200 & Cotton - O-H in plane bend \\
\hline $\begin{array}{l}1160 \text { and } \\
1110\end{array}$ & Cotton - Ether asymmetric C-O (bridge) \\
\hline 1123 & Indigo $-\mathrm{N}-\mathrm{H}$ rocking and $\mathrm{C}-\mathrm{N}$ stretching \\
\hline 1055 & Cotton - Asymmetric in phase ring stretch \\
\hline 1025 & Cotton - C-O stretch \\
\hline 1009 & $\begin{array}{l}\text { Indigo - C-C stretching in } 6 \text { membered ring an asymmetric ring breathing } \\
\text { modes }\end{array}$ \\
\hline 900 & Cotton - Asymmetric in phase ring stretch \\
\hline 880 & Indigo - In plane deformation of 5 and 6 membered rings \\
\hline 753 & Indigo - C-H wagging and out of plane deformation of 6 membered ring \\
\hline 712 & Indigo - In plane deformation of 5 and 6 membered rings \\
\hline 697 & $\begin{array}{l}\text { Indigo - Out of plane deformation of } 5 \text { and } 5 \text { membered rings and } \mathrm{C}-\mathrm{O} \\
\text { wagging }\end{array}$ \\
\hline 662 & Cotton - unassigned \\
\hline 559 & Cotton - unassigned \\
\hline
\end{tabular}

In the pristine denim there was a carbonyl peak at $1740 \mathrm{~cm}^{-1}$, this became less intense at $10 \%$ grayscale and disappeared completely at $50 \%$ grayscale. There are a range of non-cellulosic species (such as fatty acid esters and amides, waxes and pectins) covering raw cotton fibres (Hartzell-Lawson et al., 2000). The peak at $1740 \mathrm{~cm}^{-1}$ may therefore be due to ester carbonyl. It is uncertain whether the natural species described by Hartzell-Lawson et al., will still be present after the spinning, weaving and dyeing processes, though similar species could be present in lubricants etc. 


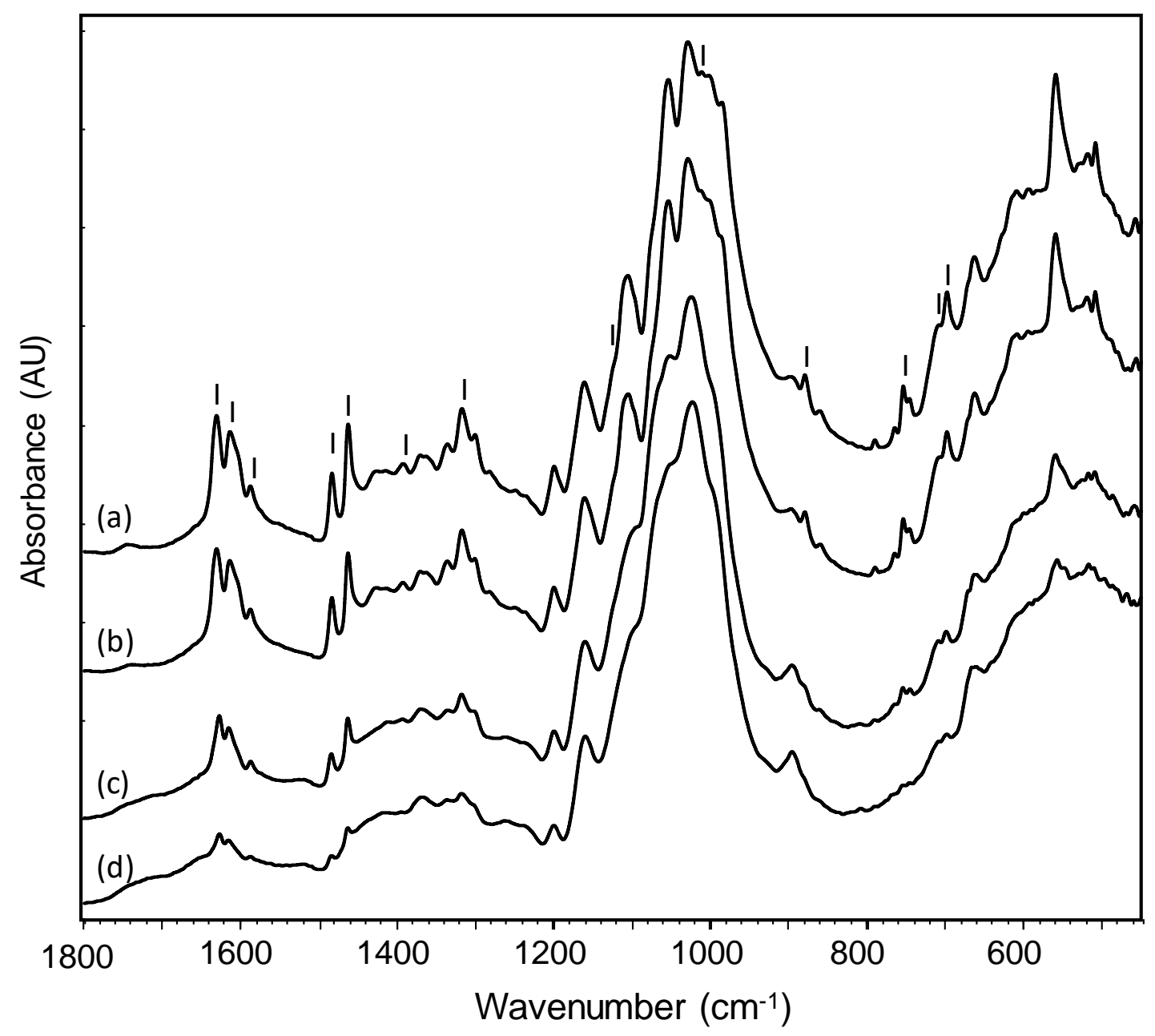

Figure 14. Stacked ATR-FTIR spectra showing the functional group and fingerprint regions for the light weight denim, 100\% laser power series; (a) pristine denim (indigo absorption bands are marked with an "I"), (b) $10 \%$ grayscale, (c) 50\% grayscale and (d) $100 \%$ grayscale.

It may nevertheless be speculated that whatever it is that is responsible for the latter carbonyl peak and the asymmetric methylene $\mathrm{C}-\mathrm{H}$ stretch at $2915 \mathrm{~cm}^{-1}$ could also have initiated thermal degradation of the denim during the OOT measurements, thereby reducing the OOT of pristine denim relative to the $10 \%$ grayscale samples.

\section{Oxidation Onset Temperature}

Oxidation onset temperature (OOT) was determined from heat flow versus time plots obtained using differential scanning calorimetry (DSC).

Graphs of OOT versus grayscale are shown in Figure 15 for all samples. It should be noted that the size of the error bars reflects a degree of heterogeneity at the DSC sample size scale (ca. 8 - $10 \mathrm{mg}$ ). Whilst statistically insignificant, the general OOT versus grayscale trends were evident across all weights and at 50 and $100 \%$ laser power. The most striking general 
observation is that a $10 \%$ grayscale the material was shown to be more stable against oxidation. This is likely to be due to removal of certain waxes and hydrocarbons (including possibly finger grease from handling) that contain groups such as carbon - carbon double bonds that are oxidised relatively easily, in the pristine ( $0 \%$ grayscale) fabric these may have initiated oxidation reactions. Subtle changes in the shape of the carbonyl bands between 0 and $10 \%$ grayscale (Figure 20) may be related to ablation of species that may initiate oxidation.

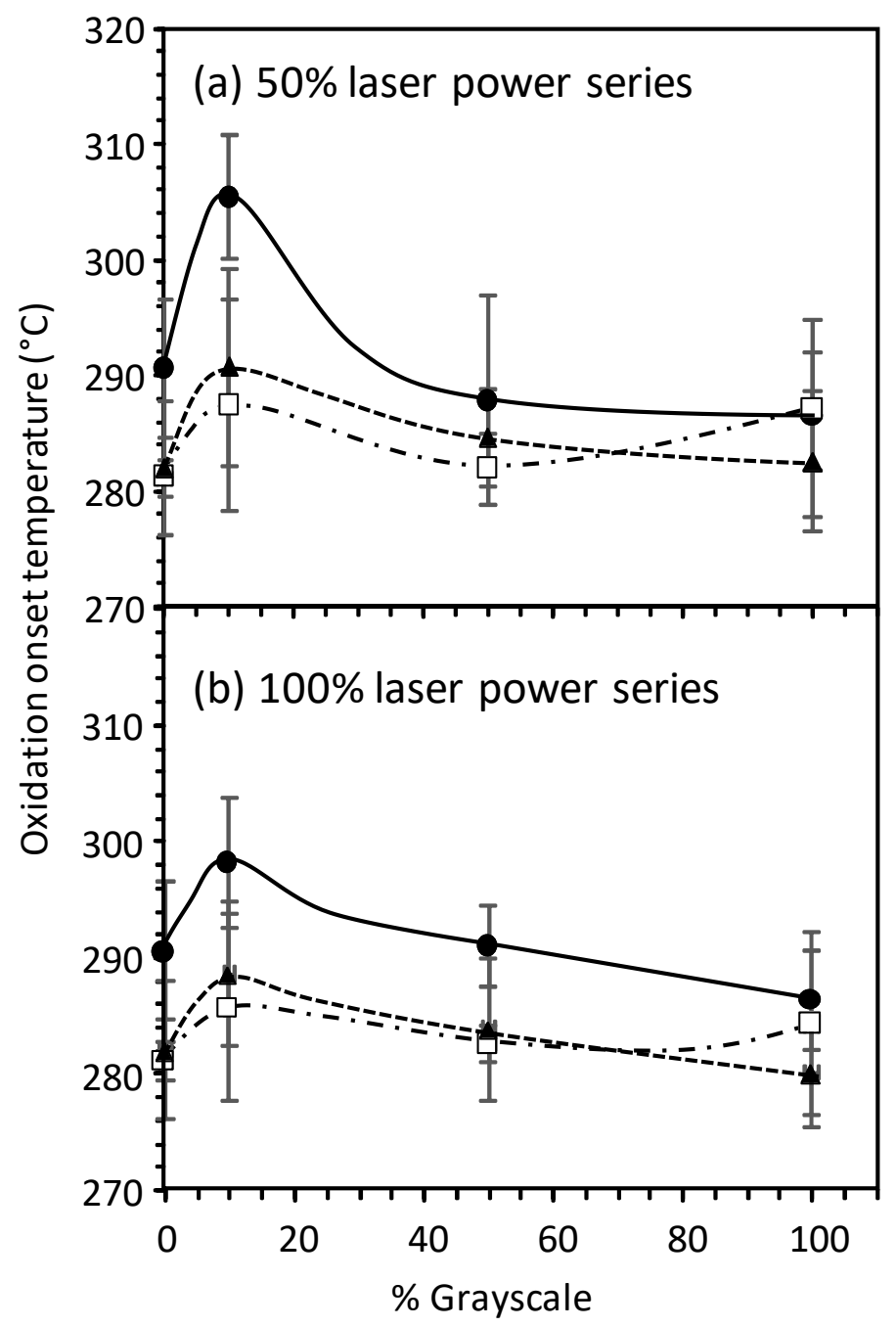

Figure 15. Average ( $n=3$ ) oxidation onset temperature (OOT) versus grayscale for; (a) samples treated with 50\% laser power and (b) samples treated with $50 \%$ laser power. The fabric weights are denoted as follows: $\boldsymbol{\Delta}$ heavy weight (solid trend line), $\square$ medium weight (dot-dashed trend line), $\bullet$ light weight (dotted trend line).

Increasing the grayscale (i.e. increasing the laser energy input) led to a general reduction in OOT due to pre-oxidation of the fabric during the laser treatment. The chemical species formed on laser treatment are likely to initiate subsequent oxidation. In some cases, samples treated at $100 \%$ laser power had reduced OOT relative to samples of equivalent grayscale treated at 
$50 \%$ laser power, due to there being a greater amount of oxidisation initiating species formed at the higher laser power. It is significant that none of the samples had OOT values that were signicantly lower than the pristine control sample.

\section{Conclusions:}

In this paper, the effect of $\mathrm{CO}_{2}$ laser engraving on denim fabric surface, structure and performance was evaluated. Colour change was investigated using spectrophotometer under two different laser power setting across three different fabric weights. In the case of colour brightness (B), it could be observed that for $50 \%$ laser power, the fabric had shown slightly higher colour brightness than $100 \%$ laser power treatment across varying grayscale, i.e. 0-100\% and this was similar across low, medium and heavy weight fabrics. However, at $100 \%$ laser power, fabric had low colour brightness, which also confirmed that dyes had been removed from the fabric surface giving it a low colour brightness. In the case of colour hue $(\mathrm{H})$, there was a consistent trend from $0-100 \%$ grayscale, where $0 \%$ grayscale had demonstrated higher blue shade, however in the case of $50 \%$ grayscale the colour hue was in the range $120-160^{\circ}$ indicating green shade and in the case of $100 \%$ grayscale shifting of indigo blue dye toward green/yellow shade was prominent. This also revealed that stepwise grayscale rating could remove colour from the fabric surface gradually and consistently. The most significant colour change was observed at $100 \%$ laser power.

The colour saturation (S) increased as the grayscale increased from 20 to $100 \%$ across $50 \%$ and $100 \%$ laser power. This trend was similar across three different fabric weights, except $0 \%$ and $10 \%$ grayscale, where higher value of saturation was noted. The spectrophotometer assessments revealed that as the grayscale increased, the lightness of the fabric increased $\left(\Delta \mathrm{L}^{*}\right)$, indicating the fabric was lighter in shade and this was consistent across LW, HW and MW fabrics. Colour change were noticed at $20 \%$ grayscale. From $\Delta \mathrm{b}^{*}$ values, it could be noted that from grayscale $30 \%-100 \%$ the fabric depicted yellow shade and $0-10 \%$ indicated fabric was blue in shade. Negative $\Delta \mathrm{a}^{*}$ values for both $50 \%$ and $100 \%$ laser power confirmed that the fabric depicted a green shade and the findings were similar to previous studies (Kan, 2014). This could be due to indigo dye reactions with the thermal energy of the laser. The reflectance curves from spectrophotometer also indicated that as the grayscale increased from $0 \%$ to $100 \%$, the depth of the shade of denim fabrics decreased indicating pale shade. A noticeable change of colour shade very different from un-etched area was evident at $30 \%$ grayscale laser etching in both 50 and $100 \%$ laser power.

SEM images confirmed that at $100 \%$ laser power the surface degradation was affected at higher grayscale compared to low grayscale. It was also interesting to note that colour change begins to occur at $20 \%$ grayscale (see Figure 4 ). The fabric structure completely degraded at high 
grayscale (>50\%) especially for LW and MW fabric, however the fabric structure in the case of HW remain intact at $100 \%$ grayscale. This can be attributed to fabric thickness and density. Laser etching at different laser power has affected fabric thickness. Fabric strength falls with decreasing fabric thickness, as the grayscale increases, this was due to formation of cellular structures of low density that had low lead bearing capacity. It was evident that fabric thickness was affected marginally more in $100 \%$ laser power than at $50 \%$ laser power. This was also noticeable across varying grayscale (0-100\%) in each fabric type.

Fabric tensile strength in warp direction was affected by laser power. For 50\% laser power, all the fabrics tends to lose its strength at $20 \%$ grayscale, in the case of LW fabric strength fell to approximately $68 \%$, however, the MW and HW fabrics remains unaffected in strength at $20 \%$ grayscale. At $100 \%$ laser power and $20 \%$ grayscale, the strength of HW, MW and LW fabrics fell to $74 \%, 57 \%$ and $37 \%$ respectively. At higher grayscale, especially beyond $50 \%$ the strength of all the fabrics were negligible. The reduction of fabric strength was due to the thermal effect produced by laser beam resulting in the loss on fibres, yarns and fabric structure. It could be inferred that $100 \%$ laser power was less suitable if fabric strength was desired especially for garment stability.

Air permeabiltiy of fabrics were less affected at $50 \%$ laser power. LW fabric showed slight decrease in air flow at $10 \%$ grayscale compared to pristine sample, and all the samples showed minor variations until 70\% grayscale; this was followed by gradual increase in air flow for LW and MW fabrics. HW fabric showed study deccrease in air flow. At 100\% laser power, due to removal of material by laser an increasing trend of air flow was observed in LW and MW fabrics and no significant change was noticed for HW fabrics. Laser engraving caused removal of materials. The SEM images of the laser etched denim (Figure 9) show degradation and fusion of the fibres, production of volatile components led to formation of gas voids within the degraded cellulose melt, leading to formation of cellular structures of reduced density and increased volume. The latter cause the fibres to fuse together resulting in a reduction in air permeability. Increased levels of degradation may lead to a greater proportion of open cell cellular structures and increased air permeability.

In the case of ATR-FTIR progressive removal (volatilisation) of the indigo dye was readily observable from the ATR-FTIR spectra, though even at $100 \%$ grayscale some indigo absorption bands remain. Damage to the cotton cellulose structure was also evident in the spectra and is most noticeable between 10 and $50 \%$ grayscale. The damage was manifested as broadening, slight blue-shifting and merging of specific inter-chain and intra-chain $\mathrm{OH}$ stretching bands, the latter together with reduction and broadening of $\mathrm{C}-\mathrm{O}$ vibrations indicates destruction of crystalline order and break-down of the cellulose polymer chains. Formation of a relatively 
small amount of non-volatile carbonyl compounds was also evident as a broad peak centred at ca. $1710 \mathrm{~cm}^{-1}$.

The DSC-OOT data obtained showed some scatter due to heterogeneity in the samples, it was however generally evident that the pristine ( $0 \%$ grayscale) samples were slightly less stable than the those engraved to $10 \%$ grayscale. This was due to removal of pro-degradant waxes and other impurities. The $50 \%$ and $100 \%$ grayscale samples however had progressively lower OOT values, though OOT did not fall substantially lower than that of the respective pristine denim samples. This may indicate that the laser engraving process does not significantly affect the potential oxidative longevity of the denim materials examined.

This study reported the colour change of denim fabrics and identified various parameters. Based on the above inferences, an optimum set of parameters (without losing the fabric performance and surface texture), where noticeable colour change can be developed between 20-30\% grayscale, $50 \%$ laser power, $100 \%$ speed, and laser pulse irradiation set at 500 pulses per inch. This can be suitable for both LW and MW fabric (0.65-0.85 mm fabric thickness). In the case of HW fabric ( $\geq 1.10 \mathrm{~mm}$, fabric thickness), $40-50 \%$ grayscale (at $50 \%$ laser power) would be ideal for developing faded effects without losing fabric structure and performance. Increases in grayscale beyond $50 \%$ (at 50\% laser power) resulted in excessive damage to the fibres that compromised the strength of the fabric. Increasing laser power to $100 \%$ led to excessive fibre damage and loss of strength, even at $20 \%$ grayscale for the LW fabric. ATR-FTIR proved effective at monitoring the decreasing amount of indigo on the cellulose fibre surfaces and increasing structural damage to the cellulose polymer, as the grayscale level increased. The laser radiation did not penetrate thickness of the fabric especially at 30-40\% grayscale and did not affect the overall fabric structure. This method of producing faded effects on denim fabric using $\mathrm{CO}_{2}$ laser at $50 \%$ power, i.e. $23 \mathrm{~W}$ (in the context of the system used in this study - laser wavelength $10.62 \mu \mathrm{m}$, pulsed wave mode) has low risks and does not cause any environmental effect compared to wet treatments and the findings are valuable to the industry.

\section{References:}

1. Adobe technical guide (2017) The HSB/HLS colour model, http://dba.med.sc.edu/price/irf/Adobe_tg/models/munsell.html; accessed online $2^{\text {nd }}$ Mar 2017

2. Baran A., Fiedler A., Schulz H., Baranska, M., (2010) In situ Raman and IR spectroscopic analysis of indigo dye, Analytical Methods, 2, pp.1373-1376

3. Bosman, J., (2007), Processes and strategies for solid state Q switch laser marking of polymers, PhD thesis, University of Twente, The Netherlands, Available from http://doc.utwente.nl/58020/1/thesis Bosmans.pdf. 
4. BS EN ISO 105 C10 (2007), Tests for colour fastness - Colour fastness to washing with soap or soap and soda, Milton Keynes: BSI, accessed online 24.03.2017

5. BS EN ISO 13934- 1 (2013), Tensile properties of fabrics. Determination of maximum force and elongation at maximum force using the strip method, Milton Keynes: BSI, accessed online 24.03.2017

6. BS EN ISO 5084: (1987), Textiles. Determination of thickness of textiles and textile products, Milton Keynes: BSI, accessed online 24.03.2017

7. BS EN ISO 9237 (1995), Determination of the permeability of fabrics to air, Milton Keynes: BSI, accessed online 24.03.2017

8. Bubonia, J.E., (2017), Colour, In: Apparel production terms and processes, $2^{\text {nd }}$ End. New York: Fairchild Books

9. Chung, C., Lee, M., and Choe, E.K., (2004) Characterisation of cotton fabric scouring by FT-IR ATR spectroscopy. Carbohydrate Polymers, 58, pp.417-420.

10. Dascalu, T., Acosta-Ortiz, S.E., Ortiz-Morates, M., and Compean, I., (2000). Removal of the indigo color by laser beam-denim interaction. Optical Laser Technology. 34, 179-189.

11. Dowden, (2009), The theory of laser materials processing: Heat Mass Transfer in Modern Technology, Berlin: Springer Science and Business Media.

12. Ferrero, F., Testore, F., Tonin C., and Innocenti, R. (2002) Surface degradation of linen textile induced by laser treatment: comparison with electron beam and heat source, Autex Research Journal, Vol 2, No.3, 109-114. http://www.autexrj.org/No5/0028.pdf

13. Garcia, B., (2015), Reduced water washing of denim garments, In: "Denim: manufacture, finishing and applications". 1st ed. Paul R., Eds: Woodhead Publishing, Elsevier Ltd

14. Ghoranneviss, M., Shahidi, S., Moazzenchi, B., Anvari, A., Rashidi, A., and Hosseini, H., (2007). Comparison between decolorization of denim fabrics with oxygen and argon glow discharge. Surface Coating Technology, 201, 4926-4930.

15. Gilbert C., Kokot S., and Meyer U., (1993) Application of DRIFT spectroscopy and chemometrics for the comparison of cotton fabrics. Applied Spectroscopy, 47(6), pp.741748.

16. Hartzell-Lawson M.M., and Hsieh Y-L (2000) Characterising non-cellulosics in developing cotton fibres. Textile Research Journal, 70(9), pp.810-819.

17. Hung, O.N., Chan, C.K., Kan, C.W., Yuen C.M.W., (2017), An analysis of some physical and chemical properties of $\mathrm{CO} 2$ laser-treated cotton-based fabrics. Cellulose, 24, pp. 363381.

18. Instructional Physics laboratory - Harvard, (2007). A summary of error propagation. Harvard University, Physical sciences 2, manual [online] Available at: http://ipl.physics.harvard.edu/wp-uploads/2013/03/PS3 Error_Propagation_sp13.pdf [Accessed 8 Jun. 2018]. 
19. International commission on illumination (2017) Commission Internationale d'Eclairage CIE, Colour Space, www.cie.co.at accessed online April 2017.

20. Jucienè, M. Urbelis, V., Juchnevičienè and Cepukonè, L. (2014). The effect of laser technological parameters on the color and structure of denim fabric. Textile Research Journal, 84(6), pp.662-670.

21. Kan, C. (2014). Colour fading effect of indigo-dyed cotton denim fabric by $\mathrm{CO}_{2}$ laser. Fibers and Polymers, 15(2), pp.426-429.

22. Kan, C. (2014a). CO2 laser treatment as a clean process for treating denim fabric. Journal of Cleaner Production, 66, pp.624-631.

23. Kan, C., Yuen, C. and Cheng, C. (2010). Technical study of the effect of $\mathrm{CO}_{2}$ laser surface engraving on the colour properties of denim fabric. Coloration Technology, 126(6), pp.365-371.

24. Kokot S., Czarnik-Matusewicz B., and Ozaki Y., (2002) Two-dimensional correlation spectroscopy and principle component analysis studies of temperature-dependent IR spectra of cotton-cellulose. Biopolymers (Biospectroscopy), 67, pp.456-469. methods for the product quality, Optics and Laser Technology, 39 (2007) 1054-1058

25. Nayak, R. and Padhye, R. (2016). The use of laser in garment manufacturing: an overview. Fashion and Textiles, 3(1).

26. Nobbs, J. H. (1985), Kubelka-Munk Theory and the Prediction of Reflectance. Review of Progress in Coloration and Related Topics, Colouration Technology, 15(1), 66-75. doi:10.1111/j.1478-4408.1985.tb03737.x

27. Nourbakhsh, S. and Yazdanshenas, M. (2008) 'Effect of corona discharge treatment on indigo dyed cotton fabric'. Coloration Technology, 124(1) pp.43-47.

28. Ondogan, Z., Pamuk, O., Ondogan, E. and Ozguney, A. (2005) 'Improving the appearance of all textile products from clothing to home textile using laser technology'. Optics \& Laser Technology, 37(8) pp.631-637.

29. Ortiz-Morales, M., Poterasu, M., Acosa-Ortiz, S., Compean, I., and Hernandez- Alvarado, M.R., (2003). A comparison between characteristics of various laser-based denim fading processes. Optics and Lasers in Engineering, 39(1), pp.15-24.

30. Ozguney, A.T., (2007), The comparison of laser surface designing and pigment printing

31. Özuguney A.T., ÖxḈelk, G. and Özkaya. K., (2009), A study on specifying the effect of laser fading process on the colour and mechanical properties of the denim fabrics, Journal of Textile and Apparel, No. 2, 133-138.

32. Paul, R. (2015) "Denim: manufacture, finishing and applications". 1st ed. 2015: Woodhead Publishing, Elsevier Ltd., pp.1-11. 
33. Štěpánková, M., Wiener, J., and Rusinová, K., (2011) Decolourization of vat dyes on cotton fabric with infrared laser light, Cellulose (2011) 18:469-478

34. Stuart, B. (2009) Infrared spectroscopy. Chichester (Royaume Uni): J. Wiley \& Sons.

35. Tarhan, M. and Sariisik, M. (2009). A Comparison Among Performance Characteristics of Various Denim Fading Processes. Textile Research Journal, 79(4), pp.301-309.

36. Textile Magazine (2016), An overview of the global and Indian denim market, Textile Magazine, 52-57.

37. Watkins, S.M., (1995), Clothing: Portable Environment, $2^{\text {nd }}$ Edition, Ames: Iowa University Press.

38. Yuan G-x, Jiang, S-x, Newton, E., Fan, J-t, and Au, W-m., (2012), Application of laser treatment for fashion design, Journal of Textile Institute, 103:1. 48-54.

\section{Acknowledgements:}

The author would like to acknowledge the kind contribution from the following technical / support colleagues: Colin Stone -assistance with vector drawings; Jim Roscoe - assistance with laser engraving laser engraving; Hayley Andrews - scanning electron microscopy, Lee Harman - infra-red spectroscopy assessments, Elaine Howarth - DSC analysis; Derek Hebdon - textile testing. The authors would also like to thank Rofinor Texteis, Portugal, for supplying the denim fabrics.

\section{Funding:}

This research did not receive any funding or grant from funding agencies in the public, commercial or not-for-profit sectors. 